Article

\title{
The Influence of Karst Aquifer Mineralogy and Geochemistry on Groundwater Characteristics: West Bank, Palestine
}

\author{
Hassan Jebreen ${ }^{1, *}\left(\mathbb{D}\right.$, Andre Banning ${ }^{1}\left(\mathbb{0}\right.$, , Stefan Wohnlich $^{1}$, Andrea Niedermayr ${ }^{1}$, \\ Marwan Ghanem ${ }^{2}$ and Frank Wisotzky ${ }^{1}$ \\ 1 Hydrogeology Department, Institute of Geology, Geophysics and Mineralogy, Ruhr University Bochum, \\ Universitätsstr. 150, 44801 Bochum, Germany; andre.banning@rub.de (A.B.); stefan.wohnlich@rub.de (S.W.); \\ andrea.niedermayr@rub.de (A.N.); frank.wisotzky@rub.de (F.W.) \\ 2 Department of Geography, Birzeit University, P.O. Box 14, Ramallah, Palestine; mghanem@birzeit.edu \\ * Correspondence: hassan.jebreen@rub.de; Tel.: +49-234-322-5387
}

Received: 31 October 2018; Accepted: 7 December 2018; Published: 11 December 2018

\begin{abstract}
This work reports, for the first time, the mineralogical and geochemical characteristics of karst aquifers in the Central West Bank (CWB) catchment in Palestine. It provides an integrated study approach by correlating the geochemistry of the lithology and hydrochemical data of groundwater samples. Mineralogical analysis showed that all of the samples were dominantly composed of either calcite $\mathrm{CaCO}_{3}\left(5-100\right.$ wt. \%) or dolomite $\mathrm{CaMg}\left(\mathrm{CO}_{3}\right)_{2}(4-100 \mathrm{wt}$. \%), with minor amounts of quartz and feldspar, which is supported by the inorganic carbon content (9-13 wt. \%) and hydrochemical composition of the spring water samples. The whole-rock geochemical data indicated that the samples have low contents of trace elements and transition metals. In contrast, the concentrations of alkaline earth elements $(\mathrm{Mg}, \mathrm{Ca}, \mathrm{Sr}, \mathrm{Ba})$ and $\mathrm{Mn}$ were high in the rock and groundwater samples. Generally, the trace elements of rock samples with concentrations $>10$ ppm included Sr (17-330 ppm), Mn (17-367 ppm), Ba (2-32 ppm), W (5-37 ppm), Cr (3-23 ppm), Zn (1.7-28 ppm), V (4-23 ppm), and $\mathrm{Zr}$ (1-22 ppm), while the concentrations of all the other trace elements was below $10 \mathrm{ppm}$. Ionic ratios and hierarchical cluster analysis (HCA) suggested that the chemical evolution of groundwater was mainly related to the geogenic (rock-water) interaction in the study area. This is clear in the alkaline earth elements $(\mathrm{Mg}, \mathrm{Ca}, \mathrm{Sr}, \mathrm{Ba})$ ratios, especially regarding the $\mathrm{Sr}$ values. The calcite rock samples had higher Sr (mean $160 \mathrm{ppm}, n=11$ ) than those of the dolomite rocks (mean $76 \mathrm{ppm}, n=9$ ).
\end{abstract}

Keywords: karst aquifer; mineralogy; geochemistry; groundwater; sediments; West Bank

\section{Introduction}

The nature and composition of the bedrock is the main control of regional groundwater mineralization. The hydrogeochemical signatures of groundwater resources are chiefly influenced by the characteristics of their catchment area. Several factors such as the climatic conditions, bedrock geology, soil properties, and anthropogenic activities influence water flow and its overall quality status [1]. The structure of karst systems is complex, and the related functioning mechanism of aquifers can be highly heterogeneous [2]. Knowledge of the properties of these karst rocks is increasingly important for the effective management of groundwater reserves and an understanding of the properties that influence a range of geotechnical issues [3]. The hydrogeochemistry of carbonate aquifers is determined by several complex processes; the most important is the dissolution and precipitation of minerals, the infiltration of surface water, biogeochemical processes, and water-rock interactions such as cation exchange, which all change the composition of groundwater [4]. 
The essential groundwater in West Bank is usually found in karstified limestone and dolomite aquifer systems. For sustainable groundwater management in West Bank aquifers, knowledge regarding the influences of the geological structure and the karst system on groundwater composition is required. As such, the western aquifer is one of the main reserves of fresh water in West Bank.

As in all karst regions, these aquifers are particularly vulnerable to pollution (see, for example, [5,6]). To date, the region has been studied mainly for its hydrochemistry $[4,7,8]$; its hydrogeology and geology have also been investigated [9-11]. Although the recharge and flow systems were investigated [12-19], no detailed mineralogical and geochemical study to investigate the composition and geological evolution of the sediments has been carried out. The current study characterizes for the first time the elemental composition (including rare earth elements) and mineralogy of sediments in Central West Bank (CWB).

The aims of this paper are to investigate the mineralogy and geochemistry of karst aquifers in Central West Bank, clarify the geological control on groundwater quality in order to identify the natural weathering processes within the catchment, and establish a link between groundwater hydrochemistry and geological materials. For this purpose, rock and spring water samples collected in the study area were analyzed for their concentration of major ions, trace elements, rare earth elements (REE), inorganic carbon content, and X-ray diffraction patterns.

\section{Materials and Methods}

\subsection{Study Site Description}

Our study area is located in the CWB, Palestine. The larger part of the study area is located within the Western Aquifer Basin (WAB), which is the largest shared groundwater aquifer between Israel and West Bank; the other part is located in the Eastern Aquifer Basin (EAB) (Figure 1). The study area lies within $35^{\circ} 00^{\prime}$ and $35^{\circ} 15^{\prime}$ longitude and $31^{\circ} 80^{\prime}$ and $32^{\circ} 10^{\prime}$ latitude, which covers about $917 \mathrm{~km}^{2}$.

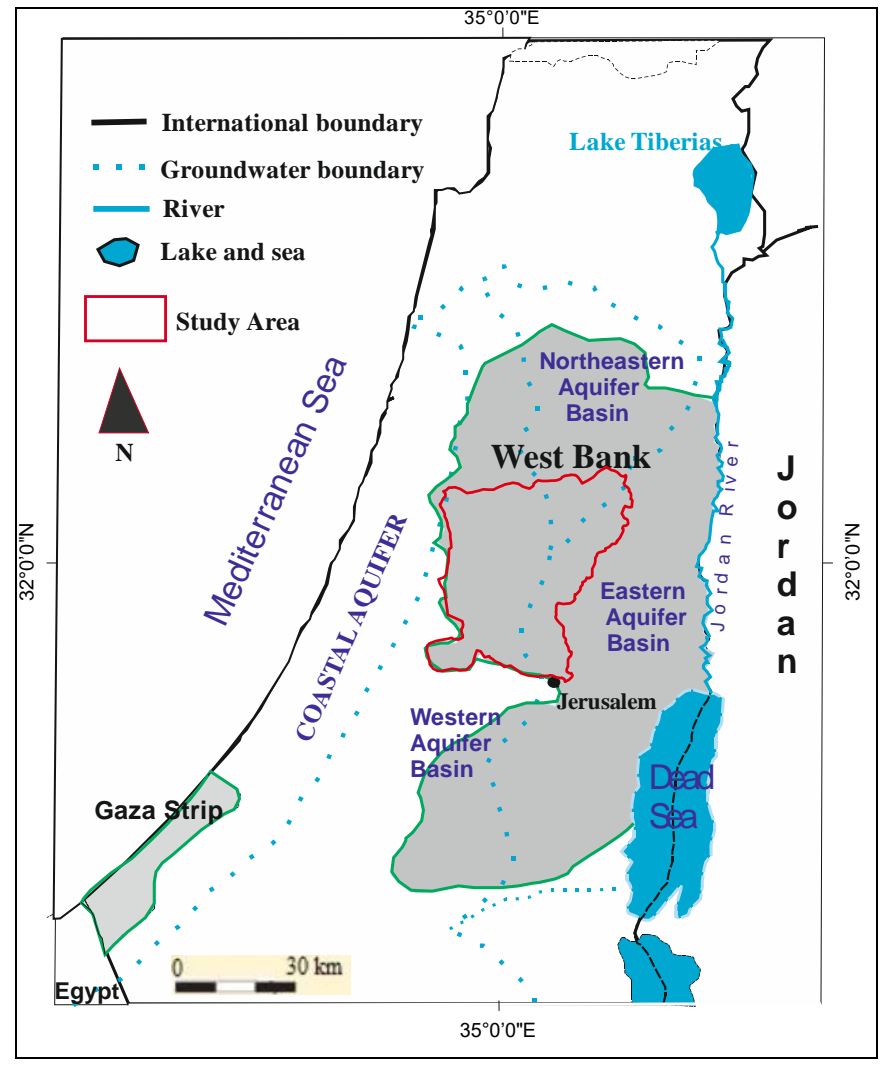

Figure 1. Location of the study area. 


\section{Geological and Hydrogeological Setting}

Climate in the recharge area is Mediterranean and semi-arid with an average precipitation of $550-700 \mathrm{~mm} /$ year over the winter [20]. Over $70 \%$ of the annual rainfall occurs between December and February. The study area is located within the recharge area of the fractured carbonate Western Mountain aquifer (Yarkon-Taninim). This is a fresh water aquifer mainly used for the production of drinking water. The estimated recharge ranges between 111-211 mm/year, representing $19 \%$ to $37 \%$ of the long-term mean annual rainfall. In addition, the mean annual actual evapotranspiration was about $66-70 \%$ of precipitation [12]. The topographic gradient from the mountain range (highest elevation: 975 m.a.s.l.) in the eastern edge of the catchment in Ramallah Mountains descends drastically to about $0.1 \mathrm{~m}$ a.s.l. in the west at the confluence with the coastal aquifer. The local geology of the study area is composed of karstic and permeable limestones and dolomites with much smaller amounts of marl and chalk interbedded with argillaceous beds of late Albian-Turonian age [21] (Figure 2).

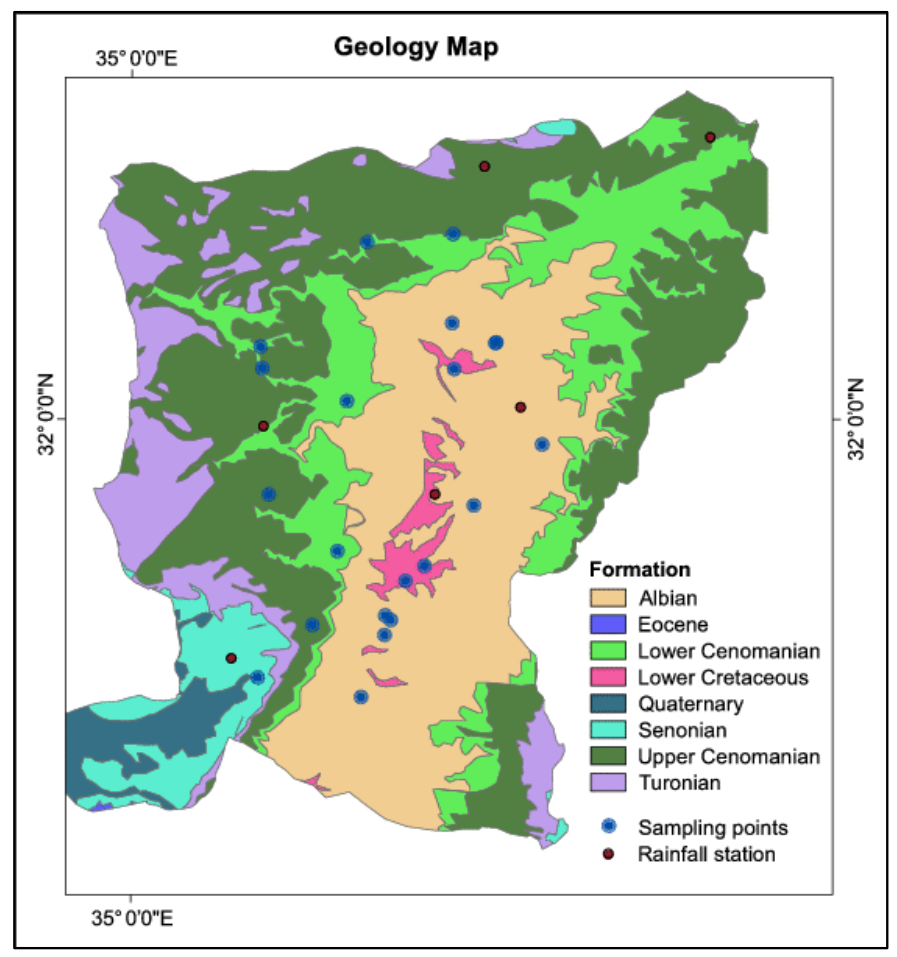

Figure 2. Geology map of the study area [12].

However, much of the carbonates contain marls, which inhibit extensive karst development, promoting the dominance of fluviokarst. Consequently, in most of the mountain regions, karstic rocks crop out, and their degree of karstification depends mainly on local climate, topography, and the amount of clay within the carbonate rocks [22]. The groundwater level is deep, ranging from a few dozen meters to 200 meters below ground level. The study area is divided into two main subaquifers (i.e., upper and lower) separated by a lower permeability layer (i.e., Yatta formation). The upper and lower subaquifer rocks are mainly composed of a sequence of hard, karstic, and permeable limestone and dolomite $[9,23]$. The hydrogeological block diagram illustrating the main geological formations is shown in Figure 3.

1. Lower aquifer: Albian-age Beit Kahil formation with 340-m thickness. This formation is found in many places in the north and the middle of the West Bank.

2. Separating layer: Cenomanian-age Yatta formation with an average thickness of about $110 \mathrm{~m}$.

3. Upper aquifer: Turonian-age Jerusalem, Bethlehem, and Hebron formations with an average thickness of $150 \mathrm{~m}$. 


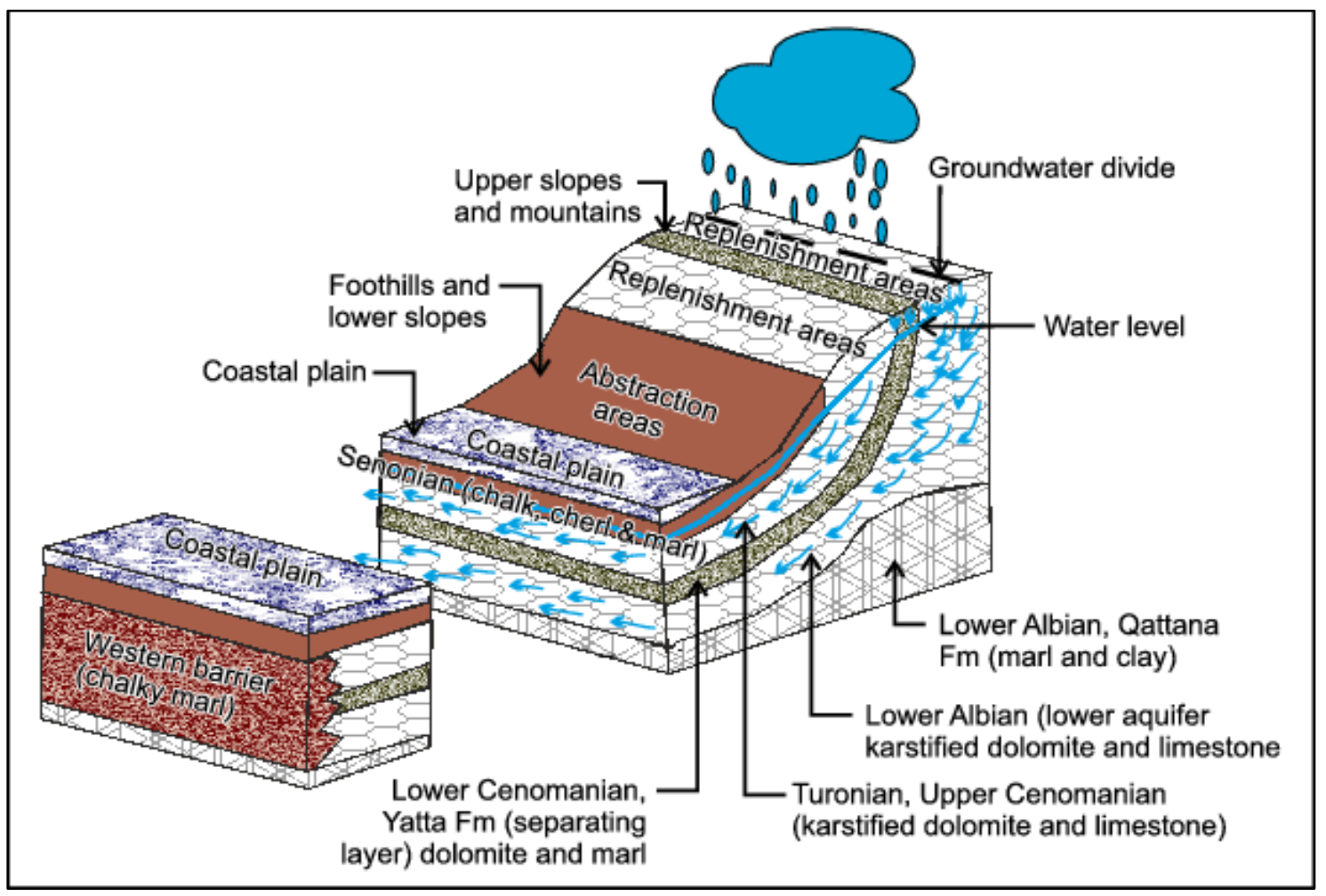

Figure 3. The hydrogeological setting of the Western Aquifer Basin (WAB) aquifer [12].

Five formations, namely the Lower Beit Kahil, Upper Beit Kahil (UBK2), Hebron, Upper Bethlehem, and Jerusalem, are comprised of pure limestone, which had partially or fully recrystallized to dolomite soon after deposition. Lower Yatta and Upper Beit Kahil (UBK1) formations are comprised of dolomites interbedded with marls. Upper Yatta Formation comprises a marly layer [4]. All of these formations are exposed over almost the entire mountain area, and lie in conformity one above the other. The chalky Lower Bethlehem formation was deposited within local troughs and basins; thus, its thickness is considerably varying between $0-150 \mathrm{~m}$, and its facies shifts in places to chalky limestone. This formation is karstified, and its groundwater is discharged by a number of springs. The lithostratigraphy column of the Central West Bank is shown in Figure 4.

\subsection{Sampling and Laboratory Analysis of Sediment}

A total of 20 rock samples were obtained from studied springs in the CWB (Figure 2). All of the samples were analyzed for bulk rock geochemistry (contents of 55 major and trace elements) by using instrumental neutron activation analysis (INAA, thermal neutron flux: $7 \times 10^{12} \mathrm{n} \mathrm{cm}^{-2} \mathrm{~s}^{-1}$, Ge detector: resolution better than $1.7 \mathrm{keV}$ for the $1332 \mathrm{keV},{ }^{60} \mathrm{Co}$ photopeak). First, $0.25 \mathrm{~g}$ of powdered sample (mesh size: 200 micros) was mixed with $\mathrm{HClO}_{4}-\mathrm{HNO}_{3}-\mathrm{HCl}-\mathrm{HF}$ acids (all analytical grade) and digested at $260{ }^{\circ} \mathrm{C}$, which was subsequently made up with diluted $\mathrm{HCl}(0.1 \mathrm{M})$. Internal quality checks were done with a frequency of $20 \%$, and sample replicates were done with analytical blanks at regular intervals. The metal concentrations were determined using inductively coupled plasma-mass spectrometry (ICP-MS). Geochemical standard reference materials (SRMs: GXR-1; GXR-4) were run concurrently with the samples. The recoveries were between $83.11-110.5 \%$ and $87.26-107.4 \%$ for trace metals. These analyses were performed by Activation Laboratories Ltd., Ancaster, Ontario/Canada. Based on the geochemical results, as well as the macroscopic rock identification, 20 samples were selected for XRD analyses to characterize their mineralogical composition. These were ground to powder-grain size in a tungsten carbide mill, before measurement by X-ray diffractometry (XRD) was applied to identify and semi-quantify the mineralogical composition of the powdered sample using 
PANalytical Empyrean instrumentation $\left(\mathrm{Cu}-\mathrm{k} \alpha, 2-65^{\circ} 2 \theta\right.$ range, $0.1^{\circ}$ step size, $10-\mathrm{s}$ counting time, operation at $45 \mathrm{kV}$, and 40-mA measurements conducted at the Institute of Geology, Mineralogy and Geophysics, Ruhr University Bochum).

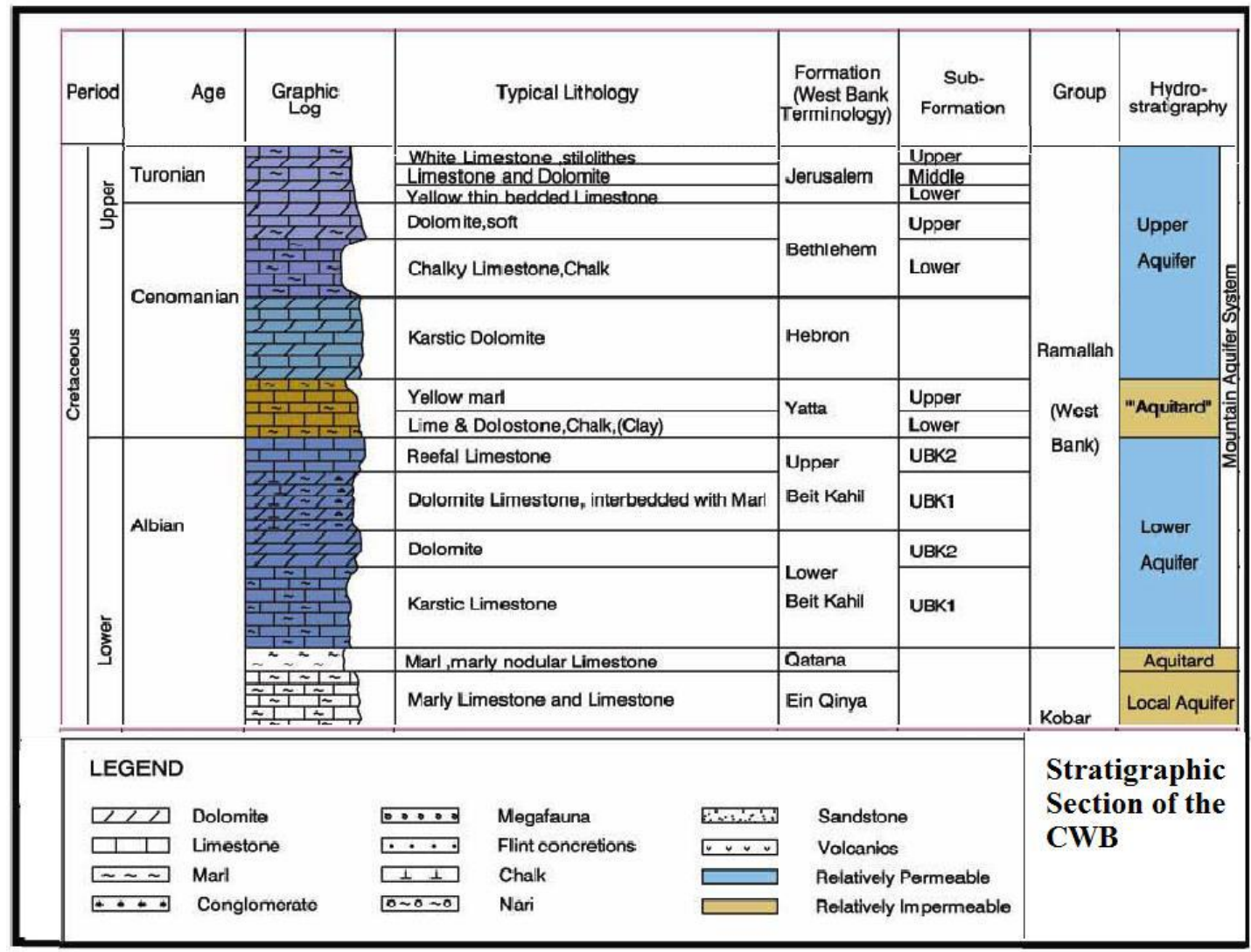

Figure 4. Lithostratigraphy column of the WAB [12].

Further aliquots of the samples were analyzed for carbon $\left(\mathrm{C}_{\text {org }} / \mathrm{C}_{\text {inorg }}\right)$ and sulfur $\left(\mathrm{S}_{\text {total }} / \mathrm{S}_{\text {pyrite }}\right)$ contents in a combustion analyzer (G4 ICARUS HF, Bruker, Billerica, MA, USA).

The contents of inorganic and organic carbon $\left(C_{\text {org }}\right.$ and $\left.C_{\text {inorg }}\right)$, as well as total and pyrite sulfur $\left(S_{\text {tot }}\right.$ and $\left.S_{\text {pyrite }}\right)$, were quantified by sediment combustion at different temperatures, followed by nondispersive infrared (NDIR) detection of produced $\mathrm{CO}_{2}$ and $\mathrm{SO}_{2}$, respectively (Bruker G4 Icarus $\mathrm{HF}$, LOD: $0.01 \mathrm{wt}$ \% $\%$ for all parameters). The normalization of REE concentration to a shale composite was done using the North American Shale Composite (NASC) [24]. This procedure allows for identifying sedimentary REE patterns through an enrichment or depletion (fractionation process) of a single element or group of REE.

\subsection{Hydrochemical Analysis}

Field campaigns also involved the collection of 20 spring water samples from 17 communities in the CWB. First, one-liter high-density polyethylene bottles were soaked with $1: 1 \mathrm{HNO}_{3}$ and washed using detergent. Water sampling bottles were properly rinsed several times with distilled water and dried in the laboratory in order to avoid contamination. Spring water samples were collected directly at their discharge under atmospheric pressure. The collected water was immediately filtered through $0.45 \mu \mathrm{m}$ membrane filters. One duplicate sample was collected at its natural $\mathrm{pH}$ in polyethylene bottles (not acidified) for anion analysis. The second sample was also collected in polyethylene bottles and acidified using $\mathrm{HNO}_{3}$ to $\mathrm{pH}<2$ for cation analysis. Collected water samples were capped gas-tight and preserved at $\sim 4{ }^{\circ} \mathrm{C}$ in a cooler container with ice blocks. Concentrations of major cations $\left(\mathrm{Ca}^{2+}, \mathrm{Mg}^{2+}\right.$, $\mathrm{Na}^{+}$, and $\mathrm{K}^{+}$) were determined by ICP-MS (Agilent Technologies 7500 Series). Trace elements also were 
analyzed by ICP-MS, and each sample was prepared by the dilution of $1.0 \mathrm{~mL}$ of the water samples to $10.0 \mathrm{~mL}$ with $0.3 \%$ ultrapure nitric acid after filtration. Each sample was analyzed three times, and the results were expressed as mean \pm standard deviation (SD). The relative standard deviation (RSD) of the three results was calculated and found to be less than $5 \%$ for all of the samples for all of the elements analyzed in this study, reflecting the precision of the method for the analysis of these trace elements [25]. All of the chemical analyses were carried out at the Aquatic and Aquaculture Research Laboratory and Environmental Research Lab at Al-Quds University in Palestine.

\subsection{Multivariate Statistical Analysis}

All data were subjected to multivariate statistical analyses (MSA) using the Statistical Package for Social Science (SPSS 17.0) software. Correlation matrix and hierarchical cluster analysis (HCA) were used. Multivariate statistical analysis broadly refers to the various statistical techniques that can be used to analyze two or more variables simultaneously.

\section{Results and Discussion}

\subsection{Mineralogical Characterization}

Interactions between groundwater and surrounding host rocks are believed to be the main processes responsible for the observed chemical characteristics of groundwater in the CWB. The evaluation of such processes requires the description of the main mineral assemblage of the rocks in which water was found, and identification of the chemical reactions that are responsible for the geochemical evolution of groundwater [26]. Mineral peak angle assignments are presented in Figure 5. Quantitative XRD analyses indicated that all of the samples were dominantly composed of either calcite $\mathrm{CaCO}_{3}$ or dolomite $\mathrm{CaMg}\left(\mathrm{CO}_{3}\right)_{2}$, with minor amounts of quartz and feldspar (Table 1), which was supported by inorganic carbon content (9-13 wt. \%). $S_{\text {pyrite }}$ as well as $S_{\text {total }}$ values were negligible (Table 1).
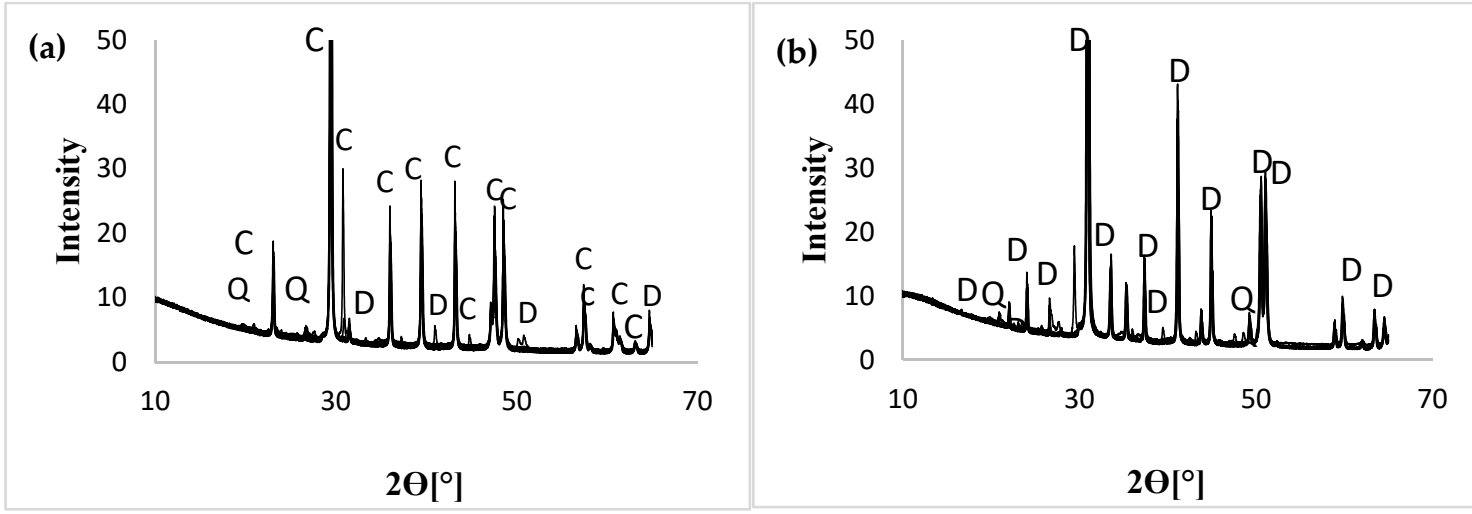

Figure 5. X-ray diffractograms. Different peak positions and heights display changes in mineralogical composition. (a) Calcite $(C)$ rock samples $(n=11)$; (b) Dolomite (D) rock samples $(n=9)$.

\subsection{Geochemistry}

Table 2 summarizes the results of the minor and trace elements analyses of rock samples. Their average concentrations in the upper crust (UC) after [27] are also listed for comparison. Generally, the trace elements with concentrations > 10 ppm include Sr (17-330 ppm), Mn (17-367 ppm), Ba (2-32 ppm), W (5-37 ppm), Cr (3-23 ppm), Zn (1.7-28 ppm), V (4-23 ppm), and Zr (1-22 ppm), while the concentration of all the other trace elements was below $10 \mathrm{ppm}$. In general, the trace element concentrations in CWB were generally lower than those of the UC, except for $\mathrm{Ca}, \mathrm{Mg}, \mathrm{Cd}, \mathrm{Mo}, \mathrm{As}$, $\mathrm{Au}, \mathrm{In}$, and Ir. Based on the mineralogy analyses, the geochemistry parameters were divided into two main groups: dolomite and calcite. 
Table 1. Quantitative XRD results, carbon/sulfur measurements, and geological formations.

\begin{tabular}{|c|c|c|c|c|c|c|c|c|c|c|c|}
\hline $\begin{array}{l}\text { Sample } \\
\text { (ID) }\end{array}$ & $\begin{array}{l}\text { Calcite } \\
\text { (C) } \\
\approx \text { wt. \% }\end{array}$ & $\begin{array}{l}\text { Dolomite } \\
\quad \text { (D) } \\
\approx \text { wt. } \%\end{array}$ & $\begin{array}{c}\text { Quartz } \\
\text { (Q) } \\
\approx \text { wt. \% }\end{array}$ & $\begin{array}{l}\text { Feldspar } \\
\approx(\mathrm{Kf})\end{array}$ & $\begin{array}{l}S_{\text {pyrite }} \\
\text { wt. \% }\end{array}$ & $\begin{array}{l}\mathrm{S}_{\text {total }} \\
\text { wt. \% }\end{array}$ & $\begin{array}{l}C_{\text {inorg }} \\
\text { wt. \% }\end{array}$ & $\begin{array}{l}\mathrm{C}_{\text {total }} \\
\text { wt. \% }\end{array}$ & Aquifer & Formation & Sub-Formation \\
\hline BA/085 & 94 & 4 & 2 & n.d. & $<0.01$ & 0.04 & 10.8 & 11.4 & Lower Cenomanian & Bethlehem & Lower \\
\hline BA $/ 085 \mathrm{~A}$ & n.d. & 100 & $<1$ & n.d. & $<0.01$ & 0.02 & 12.1 & 12.8 & Lower Cenomanian & Yatta & Lower \\
\hline $\mathrm{BA} / 108$ & $<1$ & 100 & $<<1$ & n.d. & $<0.01$ & 0.02 & 12.5 & 12.9 & Al bain & Lower Beit Kahil & UBK2 \\
\hline $\mathrm{BA} / 109$ & n.d. & 97 & 1 & 2 & $<0.01$ & 0.02 & 11.1 & 11.5 & Al bain & Lower Beit Kahil & UBK2 \\
\hline $\mathrm{BA} / 110$ & n.d. & 100 & 1 & n.d. & $<0.01$ & 0.02 & 12.5 & 13.1 & Al bain & Lower Beit Kahil & UBK2 \\
\hline $\mathrm{BA} / 111$ & 99 & n.d. & 1 & n.d. & $<0.01$ & 0.04 & 11.9 & 12.1 & Al bain & Lower Beit Kahil & UBK1 \\
\hline $\mathrm{BA} / 112$ & 100 & $<1$ & n.d. & n.d. & $<0.01$ & 0.05 & 11.1 & 12.1 & Al bain & Lower Beit Kahil & UBK1 \\
\hline $\mathrm{BA} / 117$ & n.d. & 98 & 2 & n.d. & $<0.01$ & 0.02 & 12.9 & 14.0 & Lower Cenomanian & Yatta & Lower \\
\hline $\mathrm{BA} / 121$ & n.d. & 100 & 1 & n.d. & $<0.01$ & 0.04 & 12.1 & 13.0 & Lower Cenomanian & Yatta & Lower \\
\hline $\mathrm{BA} / 122$ & 100 & n.d. & 1 & n.d. & $<0.01$ & 0.02 & 11.7 & 12.1 & Lower Cenomanian & Bethlehem & Lower \\
\hline $\mathrm{BA} / 163$ & 82 & 18 & n.d. & n.d. & $<0.01$ & 0.04 & 11.8 & 12.3 & Al bain & Lower Beit Kahil & UBK1 \\
\hline $\mathrm{BA} / 164$ & 100 & n.d. & 1 & n.d. & $<0.01$ & 0.04 & 11.7 & 12.1 & Al bain & Lower Beit Kahil & UBK1 \\
\hline $\mathrm{BA} / 170$ & 99 & n.d. & 1 & n.d. & $<0.01$ & 0.06 & 9.70 & 10.5 & Al bain & Lower Beit Kahil & UBK1 \\
\hline $\mathrm{BA} / 171$ & 100 & n.d. & n.d. & n.d. & $<0.01$ & 0.04 & 11.5 & 12.2 & Al bain & Lower Beit Kahil & UBK2 \\
\hline BA/172 & 98 & n.d. & 2 & 1 & $<0.01$ & 0.08 & 10.9 & 11.4 & Al bain & Ein Qinya & - \\
\hline NS/041 & 5 & 95 & n.d. & n.d. & $<0.01$ & 0.02 & 12.1 & 13.0 & Lower Cenomanian & Yatta & Lower \\
\hline NS/007 & n.d. & 98 & 2 & n.d. & $<0.01$ & 0.02 & 10.8 & 10.9 & Lower Cenomanian & Bethlehem & Upper \\
\hline NS/045 & 100 & n.d. & 1 & n.d. & $<0.01$ & 0.05 & 11.3 & 11.6 & Al bain & Ein Qinya & - \\
\hline Ns/02 & 100 & n.d. & 1 & n.d. & $<0.01$ & 0.02 & 11.4 & 12.1 & Upper Cenomanian & Bethlehem & Upper \\
\hline $\mathrm{Ns} / 01$ & n.d. & 96 & 4 & n.d. & $<0.01$ & 0.05 & 11.8 & 12.3 & Upper Cenomanian & Bethlehem & Upper \\
\hline
\end{tabular}

n.d. not detected. 
Table 2. Trace elements analyses of rock samples.

\begin{tabular}{|c|c|c|c|c|c|c|c|c|c|c|}
\hline \multirow{2}{*}{ Elements (ppm) } & \multirow{2}{*}{ Unit } & \multicolumn{4}{|c|}{ Dolomite Aquifers $(n=9)$} & \multicolumn{4}{|c|}{ Calcite Aquifers ( $n=11$ ) } & \multirow{2}{*}{$\begin{array}{c}\text { Upper Crust } \\
{[27]}\end{array}$} \\
\hline & & Min & Max & Mean & St dev & Min & Max & Mean & St dev & \\
\hline $\mathrm{Au}$ & $\mathrm{ppb}$ & 2.0 & 2.0 & 2.0 & 0 & 2.0 & 2.0 & 2.0 & 0 & 1.8 \\
\hline $\mathrm{Ag}$ & ppm & 0.05 & 0.05 & 0.05 & 0 & 0.06 & 0.05 & 0.051 & 0.002 & 0.05 \\
\hline $\mathrm{Cu}$ & ppm & 0.90 & 8.10 & 3.80 & 2.4 & 0.8 & 53.9 & 8.78 & 15.1 & 25 \\
\hline $\mathrm{Cd}$ & ppm & 0.10 & 0.10 & 0.10 & 0 & 0.1 & 0.40 & 0.18 & 0.11 & 0.05 \\
\hline Mo & ppm & 1.0 & 7.0 & 1.70 & 1.90 & 1 & 1.60 & 1.06 & 0.18 & 1.50 \\
\hline $\mathrm{Pb}$ & ppm & 0.50 & 1.20 & 0.70 & 0.20 & 0.50 & 1.90 & 0.82 & 0.42 & 20.0 \\
\hline $\mathrm{Ni}$ & ppm & 0.50 & 10.3 & 2.90 & 3.20 & 0.50 & 9.90 & 3.08 & 3.11 & 20.0 \\
\hline $\mathrm{Zn}$ & $\mathrm{ppm}$ & 2.30 & 7.90 & 4.34 & 2.10 & 1.70 & 28.4 & 9.37 & 9.27 & 71.0 \\
\hline $\mathrm{Al}$ & wt. \% & 0.06 & 0.76 & 0.20 & 0.22 & 0.03 & 0.9 & 0.35 & 0.25 & 8.04 \\
\hline As & ppm & 0.5 & 5.9 & 1.66 & 1.89 & 0.5 & 3 & 1.77 & 0.93 & 1.5 \\
\hline $\mathrm{Ba}$ & $\mathrm{ppm}$ & 3 & 32 & 8.55 & 9.05 & 2 & 12 & 6.81 & 3.42 & 550 \\
\hline $\mathrm{Be}$ & ppm & 0.1 & 0.1 & 0.1 & 0 & 0.1 & 0.2 & 0.124 & 0.03 & 3.0 \\
\hline $\mathrm{Bi}$ & ppm & 0.1 & 0.16 & 0.10 & 0.02 & 0.1 & 0.17 & 0.108 & 0.021 & 0.127 \\
\hline $\mathrm{Ca}$ & wt. \% & 16.3 & 19.4 & 17.9 & 0.83 & 28.1 & 31.3 & 29.7 & 1.05 & 3.0 \\
\hline Co & ppm & 2.2 & 6 & 3.55 & 1.13 & 1.3 & 7.7 & 3.47 & 2.28 & 16.0 \\
\hline $\mathrm{Cr}$ & ppm & 3 & 10 & 5.11 & 2.71 & 4 & 23 & 10.09 & 6.23 & 80.3 \\
\hline Cs & ppm & 0.05 & 0.53 & 0.17 & 0.15 & 0.05 & 0.5 & 0.20 & 0.146 & 3.7 \\
\hline $\mathrm{Fe}$ & wt. \% & 0.01 & 0.99 & 0.22 & 0.30 & 0.01 & 0.86 & 0.34 & 0.24 & 3.5 \\
\hline Hf & ppm & 0.1 & 0.6 & 0.166 & 0.164 & 0.1 & 0.3 & 0.15 & 0.09 & 5.8 \\
\hline $\mathrm{Ga}$ & ppm & 0.2 & 1.3 & 0.53 & 0.38 & 0.2 & 2.5 & 0.92 & 0.68 & 17 \\
\hline $\mathrm{Ge}$ & ppm & 0.1 & 0.16 & 0.106 & 0.02 & 0.1 & 0.18 & 0.11 & 0.02 & 1.6 \\
\hline In & ppm & 0.1 & 0.11 & 0.10 & 0.003 & 0.1 & 0.19 & 0.11 & 0.03 & 0.05 \\
\hline Ir & $\mathrm{ppb}$ & 5 & 5.1 & 5.01 & 0.03 & 5 & 5.9 & 5.19 & 0.33 & 0.2 \\
\hline $\mathrm{K}$ & wt. \% & 0.02 & 0.71 & 0.15 & 0.22 & 0.02 & 0.83 & 0.26 & 0.24 & 2.80 \\
\hline $\mathrm{Li}$ & ppm & 1.6 & 14.9 & 4.67 & 3.96 & 0.5 & 7.2 & 2.73 & 2.07 & 20.0 \\
\hline $\mathrm{Mg}$ & wt. \% & 9.66 & 11.7 & 10.9 & 0.64 & 0.21 & 1.96 & 0.53 & 0.48 & 1.33 \\
\hline $\mathrm{Mn}$ & ppm & 31 & 367 & 85 & 106.3 & 17 & 134 & 82.2 & 40.6 & 600 \\
\hline $\mathrm{Nb}$ & ppm & 0.1 & 1.7 & 0.4 & 0.50 & 0.2 & 1.9 & 0.85 & 0.59 & 25.0 \\
\hline $\mathrm{Na}$ & wt. \% & 0.01 & 0.04 & 0.02 & 0.019 & 0.01 & 0.03 & 0.01 & 0.006 & 2.89 \\
\hline $\mathrm{P}$ & wt. \% & 0.002 & 0.055 & 0.011 & 0.116 & 0.002 & 0.014 & 0.006 & 0.003 & 0.07 \\
\hline $\mathrm{Rb}$ & ppm & 0.7 & 9.4 & 2.9 & 2.74 & 0.5 & 12.5 & 4.49 & 3.65 & 112 \\
\hline $\mathrm{Sb}$ & ppm & 0.1 & 0.3 & 0.14 & 0.07 & 0.1 & 0.3 & 0.16 & 0.08 & 0.2 \\
\hline Se & ppm & 0.1 & 0.2 & 0.12 & 0.04 & 0.1 & 0.4 & 0.30 & 0.09 & 50.0 \\
\hline $\mathrm{Sr}$ & ppm & 53.3 & 112 & 76.1 & 15.7 & 16.9 & 330 & 160.5 & 87.1 & 350 \\
\hline $\mathrm{V}$ & ppm & 5 & 21 & 10.1 & 5.39 & 3 & 23 & 12.4 & 6.20 & 60.0 \\
\hline $\mathrm{U}$ & ppm & 0.9 & 2.3 & 1.58 & 0.53 & 0.2 & 3.3 & 1.66 & 0.92 & 2.8 \\
\hline W & ppm & 12 & 35 & 18.6 & 8.87 & 5 & 37 & 10.8 & 8.82 & 2.0 \\
\hline $\mathrm{Y}$ & $\mathrm{ppm}$ & 0.4 & 1.9 & 0.81 & 0.57 & 0.3 & 3.6 & 1.50 & 0.98 & 22 \\
\hline $\mathrm{Zr}$ & ppm & 1 & 22 & 4.89 & 6.64 & 3 & 16 & 7.36 & 4.90 & 190 \\
\hline
\end{tabular}

\subsection{Rare Earth Elements}

The sum of the total REE ( $\Sigma$ REE), light REE (LREE), and heavy REE (HREE) concentrations, and the normalized $(\mathrm{La} / \mathrm{Gd})_{\mathrm{N}}$ and $(\mathrm{La} / \mathrm{Yb})_{\mathrm{N}}$ ratios of the analyzed samples, are shown in Table 3. The average REE contents of the UC are also listed for comparison. Higher contents of REE were found in calcite rocks than in dolomite rocks. The $\Sigma$ LREE values varied between 1.9-19.8 ppm (average value $6.4 \mathrm{ppm}$ ), among which the contents of $\mathrm{La}, \mathrm{Ce}$ and $\mathrm{Nd}$ were relatively high. The $\Sigma$ HREE values varied between 0.6-2 ppm, with an average of $0.8 \mathrm{ppm}$. The LREE/HREE ratio showed that the content of LREE was significantly higher than that of HREE. The variation extent of the LREE/HREE ratio was from 2.08 to 11.0 (average value 6.72), and thus featured by typical LREE enrichment and HREE deficiency, which suggested the vulnerability of HREEs to dilution in the dolomitization process [28], and also that mineralogy might play a role in controlling the distribution of REE in carbonates. All of the samples show a $(\mathrm{La} / \mathrm{Yb})_{\mathrm{N}}>1$, meaning enrichment in LREE in the CWB sediments. The ratio of $\mathrm{La} / \mathrm{Gd}_{\mathrm{N}}<1$ suggests a moderate enrichment in MREE relative to LREE. However, the CWB rock sediments were characterized by low $\sum R E E$ content (average $7.27 \mathrm{ppm}$ ). The Eu anomalies (Eu/Eu*) showed high variance ranging from 0.60 to 1.52, and most of them show weak negative Eu anomalies. Meanwhile, the $\mathrm{Ce}$ anomalies $\left(\mathrm{Ce} / \mathrm{Ce}^{*}\right)$ showed variance ranging from 0.87 to 1.12, and most of them showed positive Ce anomalies. The sediments had a lower concentration of the REE compared to shale (NASC: North American Shale Composite) (Figure 6). All of the samples had lower REE contents than those in the UC. 
Table 3. Concentration of rare earth elements (ppm).

\begin{tabular}{|c|c|c|c|c|c|c|c|c|c|c|c|c|c|c|c|c|c|c|c|c|c|}
\hline $\begin{array}{c}\text { Elements } \\
\text { (ppm) }\end{array}$ & $\begin{array}{c}\text { BA } \\
/ 085\end{array}$ & $\begin{array}{c}\text { BA } \\
/ 085 A\end{array}$ & $\begin{array}{c}\text { BA } \\
/ 108\end{array}$ & $\begin{array}{c}\text { BA } \\
/ 109\end{array}$ & $\begin{array}{c}\text { BA } \\
/ 110 \\
\end{array}$ & $\begin{array}{r}\text { BA } \\
/ 111\end{array}$ & $\begin{array}{r}\text { BA } \\
/ 112 \\
\end{array}$ & $\begin{array}{r}\text { BA } \\
/ 117\end{array}$ & $\begin{array}{r}\text { BA } \\
/ 121 \\
\end{array}$ & $\begin{array}{r}\text { BA } \\
/ 122\end{array}$ & $\begin{array}{c}\text { BA } \\
/ 163\end{array}$ & $\begin{array}{c}\text { BA } \\
/ 164\end{array}$ & $\begin{array}{c}\text { BA } \\
/ 170\end{array}$ & $\begin{array}{c}\text { BA } \\
/ 171\end{array}$ & $\begin{array}{c}\text { BA } \\
/ 172 \\
\end{array}$ & $\begin{array}{l}\text { NS } \\
/ 041\end{array}$ & $\begin{array}{l}\text { NS } \\
/ 007\end{array}$ & $\begin{array}{c}\text { NS } \\
\text { /045 }\end{array}$ & $\begin{array}{l}\text { NS } \\
/ 02\end{array}$ & $\begin{array}{l}\text { NS } \\
/ 01\end{array}$ & $\begin{array}{c}\text { Upper Crus } \\
{[27]}\end{array}$ \\
\hline $\mathrm{La}$ & 1.70 & 0.90 & 0.50 & 2.70 & 0.40 & 1.40 & 0.90 & 0.80 & 0.70 & 0.60 & 0.80 & 1.60 & 4.30 & 0.30 & 3.40 & 0.50 & 1.20 & 2.0 & 1.60 & 1.80 & 30.0 \\
\hline $\mathrm{Ce}$ & 3.30 & 1.60 & 0.70 & 5.0 & 0.80 & 2.70 & 1.80 & 1.70 & 1.20 & 1.20 & 1.60 & 3.0 & 8.40 & 0.40 & 6.50 & 0.90 & 2.40 & 4.10 & 3.10 & 3.80 & 64.0 \\
\hline $\operatorname{Pr}$ & 0.40 & 0.20 & 0.10 & 0.60 & 0.10 & 0.30 & 0.20 & 0.20 & 0.10 & 0.10 & 0.20 & 0.30 & 1.0 & 0.10 & 0.80 & 0.10 & 0.30 & 0.50 & 0.30 & 0.50 & 7.10 \\
\hline $\mathrm{Nd}$ & 1.70 & 0.70 & 0.40 & 2.50 & 0.40 & 1.30 & 0.90 & 0.70 & 0.60 & 0.60 & 0.80 & 1.60 & 4.30 & 0.20 & 3.50 & 0.40 & 1.10 & 2.10 & 1.50 & 2.0 & 26.0 \\
\hline $\mathrm{Sm}$ & 0.30 & 0.10 & 0.10 & 0.50 & 0.10 & 0.20 & 0.20 & 0.10 & 0.10 & 0.10 & 0.10 & 0.30 & 0.80 & 0.10 & 0.70 & 0.10 & 0.20 & 0.40 & 0.30 & 0.40 & 4.50 \\
\hline $\mathrm{Eu}$ & 0.06 & 0.05 & 0.05 & 0.11 & 0.05 & 0.07 & 0.05 & 0.05 & 0.05 & 0.05 & 0.05 & 0.08 & 0.21 & 0.05 & 0.17 & 0.05 & 0.05 & 0.12 & 0.08 & 0.10 & 0.88 \\
\hline $\mathrm{Gd}$ & 0.30 & 0.10 & 0.10 & 0.50 & 0.10 & 0.30 & 0.20 & 0.10 & 0.10 & 0.10 & 0.20 & 0.40 & 0.80 & 0.10 & 0.70 & 0.10 & 0.20 & 0.50 & 0.20 & 0.40 & 3.80 \\
\hline Dy & 0.20 & 0.10 & 0.10 & 0.50 & 0.10 & 0.20 & 0.20 & 0.10 & 0.10 & 0.10 & 0.10 & 0.30 & 0.70 & 0.10 & 0.60 & 0.10 & 0.20 & 0.40 & 0.30 & 0.40 & 3.50 \\
\hline Ho & 0.10 & 0.10 & 0.10 & 0.20 & 0.10 & 0.10 & 0.10 & 0.10 & 0.10 & 0.10 & 0.10 & 0.20 & 0.40 & 0.10 & 0.30 & 0.10 & 0.10 & 0.20 & 0.10 & 0.20 & 0.80 \\
\hline Er & 0.10 & 0.10 & 0.10 & 0.20 & 0.10 & 0.10 & 0.10 & 0.10 & 0.10 & 0.10 & 0.10 & 0.20 & 0.40 & 0.10 & 0.30 & 0.10 & 0.10 & 0.20 & 0.10 & 0.20 & 2.30 \\
\hline $\mathrm{Tm}$ & 0.10 & 0.10 & 0.10 & 0.10 & 0.10 & 0.10 & 0.10 & 0.10 & 0.10 & 0.10 & 0.10 & 0.10 & 0.10 & 0.10 & 0.10 & 0.10 & 0.10 & 0.10 & 0.10 & 0.10 & 0.33 \\
\hline $\mathrm{Yb}$ & 0.10 & 0.10 & 0.10 & 0.20 & 0.10 & 0.10 & 0.10 & 0.10 & 0.10 & 0.10 & 0.10 & 0.10 & 0.30 & 0.10 & 0.20 & 0.10 & 0.10 & 0.20 & 0.10 & 0.20 & 2.20 \\
\hline $\mathrm{Lu}$ & 0.10 & 0.10 & 0.10 & 0.10 & 0.10 & 0.10 & 0.10 & 0.10 & 0.10 & 0.10 & 0.10 & 0.10 & 0.10 & 0.10 & 0.10 & 0.10 & 0.10 & 0.10 & 0.10 & 0.10 & 0.32 \\
\hline$\Sigma$ REE & 8.46 & 4.25 & 2.55 & 13.21 & 2.55 & 6.97 & 4.95 & 4.25 & 3.45 & 3.35 & 4.35 & 8.28 & 21.81 & 1.85 & 17.37 & 2.75 & 6.15 & 10.92 & 7.88 & 10.2 & - \\
\hline$\Sigma$ LREE & 7.76 & 3.65 & 1.95 & 11.91 & 1.95 & 6.27 & 4.25 & 3.65 & 2.85 & 2.75 & 3.75 & 7.28 & 19.81 & 1.25 & 15.77 & 2.15 & 5.45 & 9.72 & 7.08 & 9.0 & - \\
\hline$\Sigma$ HREE & 0.70 & 0.60 & 0.60 & 1.30 & 0.60 & 0.70 & 0.70 & 0.60 & 0.60 & 0.60 & 0.60 & 1.0 & 2.0 & 0.60 & 1.60 & 0.60 & 0.70 & 1.20 & 0.80 & 1.20 & - \\
\hline LREE/HREE & 11.0 & 6.08 & 3.25 & 9.16 & 3.25 & 8.95 & 6.07 & 6.08 & 4.75 & 4.58 & 6.25 & 7.28 & 9.90 & 2.08 & 9.85 & 3.58 & 7.78 & 8.1 & 8.85 & 7.5 & - \\
\hline ¿REE & 8.46 & 4.25 & 2.55 & 13.21 & 2.55 & 6.97 & 4.95 & 4.25 & 3.45 & 3.35 & 4.35 & 8.28 & 21.81 & 1.85 & 17.37 & 2.75 & 6.15 & 10.92 & 7.88 & 10.2 & - \\
\hline $\mathrm{Ce} / \mathrm{Ce} *$ & 1.04 & 1.08 & 0.84 & 1.03 & 1.07 & 1.07 & 1.07 & 1.21 & 0.99 & 1.07 & 1.07 & 1.0 & 1.04 & 0.87 & 1.01 & 1.08 & 1.12 & 1.07 & 1.07 & 1.07 & - \\
\hline Eu/Eu* & 0.60 & 1.52 & 1.52 & 0.67 & 1.52 & 0.87 & 0.76 & 1.52 & 1.52 & 1.52 & 1.07 & 0.70 & 0.80 & 1.52 & 0.74 & 1.52 & 0.76 & 0.81 & 0.99 & 0.76 & - \\
\hline$(\mathrm{La} / \mathrm{Yb})_{\mathrm{N}}$ & 11.5 & 6.11 & 3.39 & 9.17 & 2.71 & 9.51 & 6.11 & 5.43 & 4.75 & 4.07 & 5.43 & 10.8 & 9.73 & 2.03 & 11.5 & 3.39 & 8.15 & 6.79 & 10.8 & 6.11 & - \\
\hline$(\mathrm{La} / \mathrm{Gd})_{\mathrm{N}}$ & 4.75 & 7.55 & 4.19 & 4.53 & 3.35 & 3.91 & 3.77 & 6.71 & 5.87 & 5.03 & 3.35 & 3.35 & 4.51 & 2.51 & 4.07 & 4.14 & 5.03 & 3.35 & 6.71 & 3.77 & - \\
\hline
\end{tabular}

Abbreviations: Light REE (LREE: La to Sm); heavy REE (HREE: Eu to Lu); $\Sigma$ REE = sum of total concentration of rare earth elements; Ratio $=\Sigma \mathrm{LREE} / \Sigma \mathrm{HREE}$; Ce/Ce* = the value of the Ce

anomaly calculated by the formula Ce/Ce* $=\left[\left(\mathrm{Ce}_{\mathrm{N}}\right) /\left(\mathrm{SOR}\left(\mathrm{La}_{\mathrm{N}} * \mathrm{Pr}_{\mathrm{N}}\right)\right)\right]$ from $[29] ; \mathrm{Eu} / \mathrm{Eu} *=$ the value of the Eu anomaly calculated by the formula $[\mathrm{Eu} / \mathrm{Eu} *]=\left[(\mathrm{EuN}) /\left(\mathrm{SQR}\left(\mathrm{Sm} \mathrm{N}_{\mathrm{N}}\right.\right.\right.$ *

$\left.\mathrm{Gd}_{\mathrm{N}}\right)$ )] from [29]. $\mathrm{Eu}_{\mathrm{N}}, \mathrm{Sm}_{\mathrm{N}}, \mathrm{Gd}_{\mathrm{N}}=$ shale-normalized values against NASC [24]; $(\mathrm{La} / \mathrm{Gd})_{\mathrm{N}}$ and $(\mathrm{La} / \mathrm{Yb})_{\mathrm{N}}$ are normalized concentration ratios in the selected samples. 


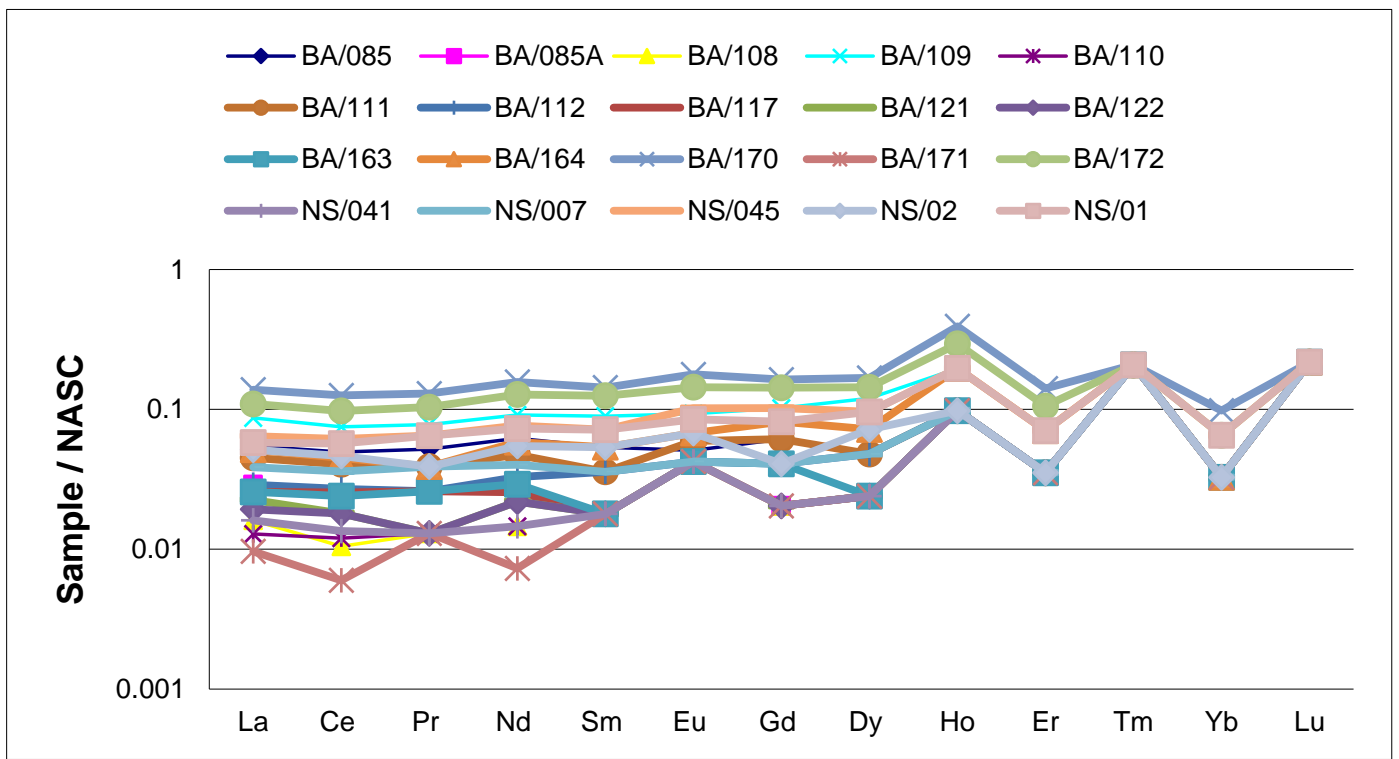

Figure 6. North American Shale Composite (NASC) normalized rare earth element (REE) patterns of the rock samples of the study area.

\subsection{Hydrochemistry}

\subsubsection{Carbonate Weathering and Dissolution}

Carbonate rocks are generally composed of two major minerals (calcite and dolomite) and their associated members (Table 4). Their composition depends on the mineralogy, partition coefficients and enrichment factor of individual elements and ion exchange processes during deposition. The carbonate minerals involved in this mechanism were calcite, aragonite, and dolomite, which contain $\mathrm{Ca}$ and $\mathrm{Mg}$. The mechanism involved in the aquifer is explained by the carbonate matrix of the reservoir, where the dissolution of calcite (Equation (1)) and dolomite (Equation (2)) in the presence of $\mathrm{CO}_{2}$ are likely the main processes of mineralization.

$$
\begin{gathered}
\mathrm{CO}_{2}+\mathrm{H}_{2} \mathrm{O}+\mathrm{CaCO}_{3} \rightarrow \mathrm{Ca}^{2+}+2 \mathrm{HCO}_{3}^{-}: \text {calcite dissolution } \\
2 \mathrm{CO}_{2}+2 \mathrm{H}_{2} \mathrm{O}+\mathrm{CaMg}\left(\mathrm{CO}_{3}\right)_{2} \rightarrow \mathrm{Ca}^{2+}+\mathrm{Mg}+4 \mathrm{HCO}_{3}^{-}: \text {dolomite dissolution }
\end{gathered}
$$

The groundwater and dolomite rock samples indicated having consistently higher molar $\mathrm{Mg} / \mathrm{Ca}$ ratios than the coexisting calcites, and were close to the dissolution trend of dolomite. The calcite rock samples had higher $\mathrm{Sr}$ (mean $160 \mathrm{ppm}, n=11$ ) values than those of the dolomite rocks (mean $76 \mathrm{ppm}, n=9$ ). The available carbonates in these rocks might have been dissolved and added to the groundwater system during irrigation, rainfall infiltration, and groundwater movement. In karst groundwater, the karst processes (dissolution of carbonate rock) lead to the release of $\mathrm{Ca}, \mathrm{Mg}$ and $\mathrm{Sr}$ into water.

Table 4. Major mineral groups and their associated members generally found in carbonate rocks [30].

\begin{tabular}{cccc}
\hline Group & $\begin{array}{c}\text { Major } \\
\text { Composition }\end{array}$ & Common Impurities & Members \\
Calcite & $\mathrm{CaCO}_{3}$ & $\begin{array}{c}\mathrm{Mn}, \mathrm{Fe}, \mathrm{Zn}, \mathrm{Co}, \mathrm{Sr}, \mathrm{Pb}, \\
\mathrm{Mg}, \mathrm{Cu}, \mathrm{Al}, \mathrm{Ni}, \mathrm{V}, \mathrm{Cr}, \mathrm{Mo}\end{array}$ & $\begin{array}{c}\left.\text { Gaspeite }[\mathrm{Ni}, \mathrm{Mg}, \mathrm{Fe}) \mathrm{CO}_{3}\right] \text {, magnesite } \\
{\left[\mathrm{MgCO}_{3}\right], \text { otavite }\left[\mathrm{CdCO}_{3}\right], \text { rhodochrosite }} \\
{\left[\mathrm{MnCO}_{3}\right], \text { siderite }\left[\mathrm{FeCO} \mathrm{CO}_{3}\right], \text { smithsonite }} \\
{[\mathrm{ZnCO}], \text { spherocobaltite }\left[\mathrm{CoCO} \mathrm{C}_{3}\right]}\end{array}$ \\
\hline Dolomite & $\mathrm{CaMg}\left(\mathrm{CO}_{3}\right)_{2}$ & $\mathrm{Fe}, \mathrm{Mn}, \mathrm{Co}, \mathrm{Pb}, \mathrm{Zn}$ & $\begin{array}{c}\text { Ankerite }\left[\mathrm{Ca}(\mathrm{Fe}, \mathrm{Mg})\left(\mathrm{CO}_{3}\right)_{2}\right], \text { kutnohorite } \\
{\left[\mathrm{Ca}(\mathrm{Mn}, \mathrm{Mg}, \mathrm{Fe})\left(\mathrm{CO}_{3}\right)_{2}\right], \text { minrecordite }} \\
{\left[\mathrm{CaZn}\left(\mathrm{CO}_{3}\right)_{2}\right], \text { norsethite }\left[\mathrm{BaMg}\left(\mathrm{CO}_{3}\right)_{2}\right]}\end{array}$ \\
\hline
\end{tabular}




\subsubsection{Major Ion Chemistry}

Data on the major ion chemical composition of the CWB spring samples were obtained and discussed from previous study [4], as there was limited information on the trace element composition of these rocks. The hydrogeochemical facies of groundwater is primarily dependent on the geology, rock-water interactions, and solution kinetics, as well as contamination sources occurring within the aquifer system. A convenient method is to classify and compare water types based on ionic composition. The distribution of cations and anions used various plots such as the trilinear Piper diagram [31]. The chemical groundwater types of the study area were distinguished and grouped by their position in a Piper diagram (Figure 7) [4].

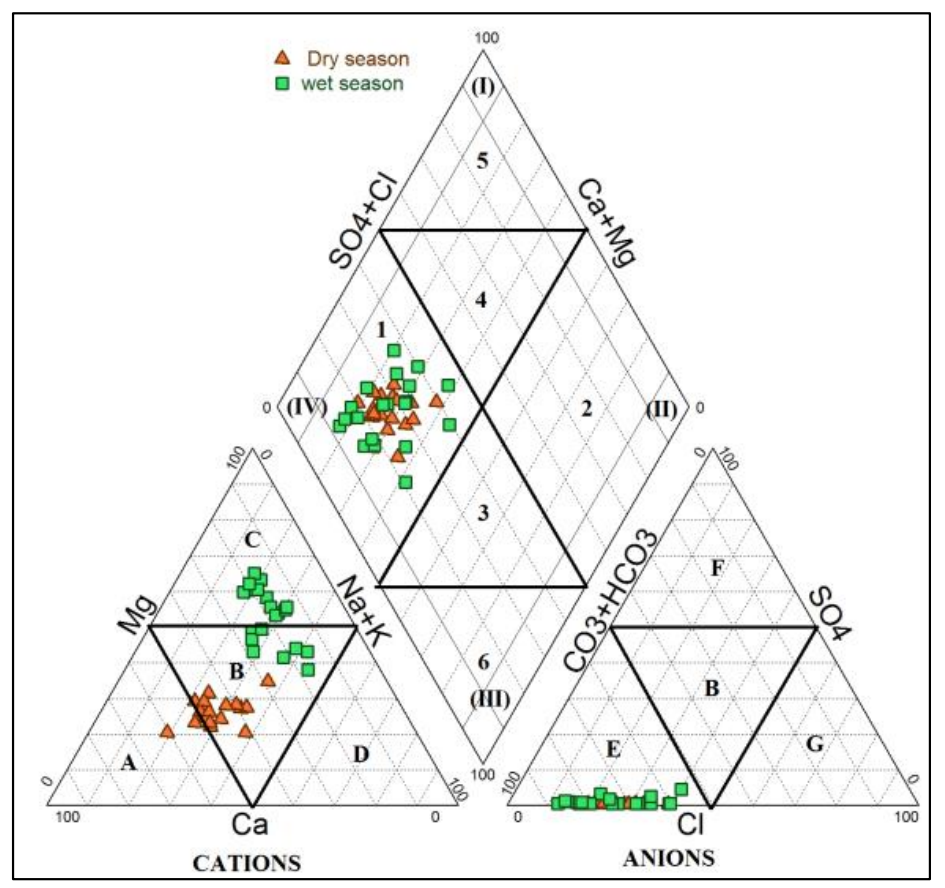

Figure 7. Piper diagram of the spring water samples in the study area (data from [4]).

One main type of water has been identified based on varying ionic concentrations: $\mathrm{Ca}-\mathrm{Mg}-\mathrm{HCO}_{3}$, which indicates that alkaline earths and weak acidic anions exceed alkali metals and strongly acidic anions; this type indicates mineral dissolution (probably carbonate minerals). The results showed that the hydrochemistry of karst water in the study area has different characteristics temporally. Within the dry season, the dominant hydrogeochemical process is water-rock interaction (precipitation and dissolution of calcite and dolomite), which is indicated by achieving an ample amount of the mineral within a sufficient residence time. Conversely, in the rainy season, the hydrogeochemical process shifted from water-rock interaction to the dilution of rainwater (Figure 8a) as a result of rain water supply through a conduit channel system, which is characterized by declining $\mathrm{Ca}^{2+}, \mathrm{HCO}_{3}{ }^{-}$, TDS, SI calcite and dolomite in the groundwater.

The dilution by rainwater also caused an increase in the correlation of some hydrogeochemical parameters. The rain will additionally transport the fertilizer from soil to the groundwater, corresponding to the vast areas in the CWB under agricultural use. This indicates that in addition to the dissolution of deposited carbonates, $\mathrm{Mg}^{2+}$ and $\mathrm{Na}^{+}$ions are added to the groundwater during the wet season relative to $\mathrm{Ca}^{2+}$, in comparison with the ratios during the dry season [4]. On the other hand, the intrusion of brine water into adjacent aquifers probably generally increases their salinity [32]. However, total concentrations during the wet season were lower, as the residence time in the aquifer was shorter, and the evaporation effects in the groundwater were smaller. The residence time and the intensity of recharge play important roles in controlling the chemical composition of the spring water in 
both seasons. Figure 8 shows an example of water-rock interaction; it can be seen that the influence of precipitation in controlling spring water chemistry is much stronger in the wet season (Figure 8a) [4].
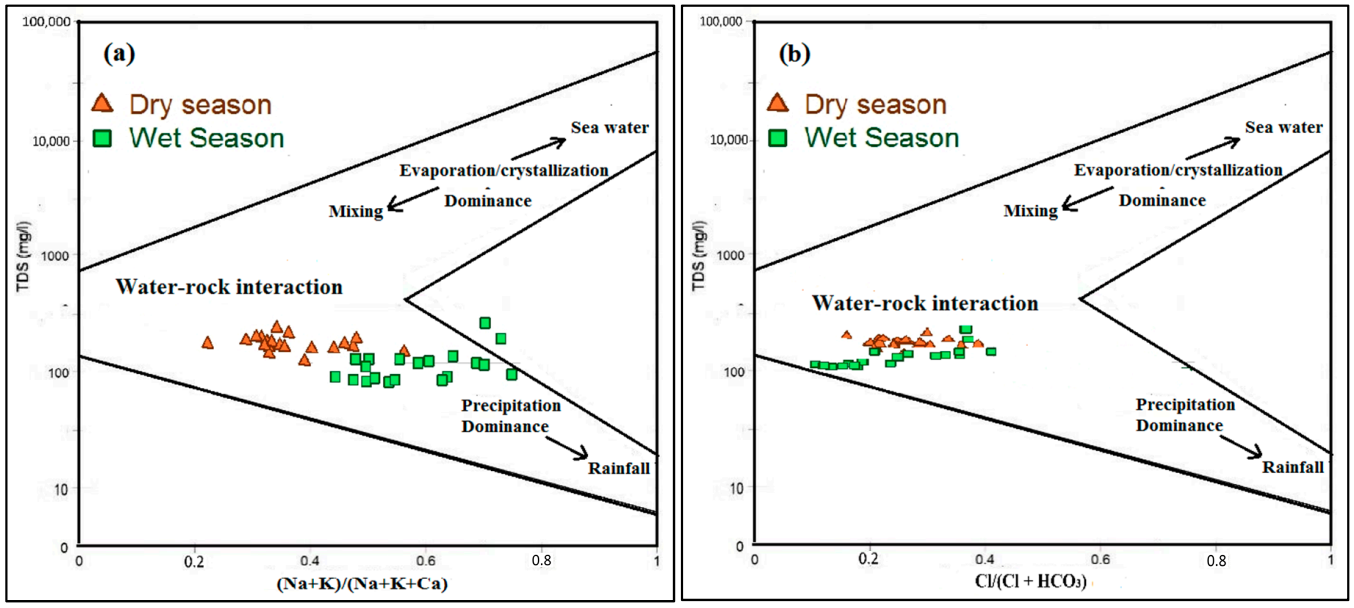

Figure 8. Gibbs graphs of the spring water sample: (a) cations; (b) anions (data from [4]).

\subsubsection{Trace Elements in Groundwater}

The statistical results of various trace elemental concentrations in the groundwater of the CWB are given in Table 5. For the present study, samples were collected from 20 different locations and analyzed for Li, B, Al, Ba, Ti, Pb, Bi, Cr, Mn, Fe, Ni, Co, Ni, Cu, Zn, Sr, Mo, Ag, and Cd. Trace element concentrations in karst water are usually low, because of short residence time. Results showed that all of the trace elements were detected in all of the spring water samples that were analyzed.

Generally, the trace elements of spring water samples with high mean values included Fe (111.1 $\mu \mathrm{g} / \mathrm{L}), \mathrm{Sr}(91.2 \mu \mathrm{g} / \mathrm{L}), \mathrm{B}(26.4 \mu \mathrm{g} / \mathrm{L}), \mathrm{Ba}(17.9 \mu \mathrm{g} / \mathrm{L}), \mathrm{Al}(17.6 \mu \mathrm{g} / \mathrm{L})$, and Zn $(4.33 \mu \mathrm{g} / \mathrm{L})$, while the mean values of all the other trace elements were below $4 \mu \mathrm{g} / \mathrm{L}$. The primary source of groundwater $\mathrm{Fe}, \mathrm{Sr}, \mathrm{B}, \mathrm{Ba}, \mathrm{Al}$, and $\mathrm{Zn}$ was likely to be the dissolution of carbonate minerals [33]. They generally possessed fairly similar mean and median values, which indicates a normal and homogeneous distribution. Alkaline earth trace metals (Ba and $\mathrm{Sr}$ ) behave geochemically similar to $\mathrm{Ca}$ and $\mathrm{Mg}$, and are therefore able to stoichiometrically substitute the primary elements in carbonates.

\subsection{Statistical Analysis}

\subsubsection{Correlation Matrix}

The Pearson correlation matrix is normally used to investigate the relationships between the various parameters of all of the hydrochemical samples in this study. The correlation matrix is helpful for determining the relationships among the variables and influencing factors, which helps identify the sources of different elements by the values highlighted in bold (Table 6).

The correlation of groundwater samples showed that a strong significant positive correlation existed between the following parameters: Ca and Fe $\left(r^{2}=0.95\right), \mathrm{Sr}\left(\mathrm{r}^{2}=0.87\right), \mathrm{Ba}\left(\mathrm{r}^{2}=0.86\right), \mathrm{Mg}$ $\left(r^{2}=0.80\right), B\left(r^{2}=0.71\right)$, and $\mathrm{Cu}\left(r^{2}=0.66\right)$. Sr is a common constituent of carbonate minerals, and is likely to have been derived from their dissolution, which contributes about $15-28 \%$ to the total chemical input of $\mathrm{Sr}$ to groundwater from karst aquifers. Strontium in groundwater was positively correlated with Fe $\left(r^{2}=0.89\right), \mathrm{Ca}\left(\mathrm{r}^{2}=0.87\right), \mathrm{Ba}\left(\mathrm{r}^{2}=0.86\right), \mathrm{Li}\left(\mathrm{r}^{2}=0.73\right), \mathrm{Cu}\left(\mathrm{r}^{2}=0.69\right), \mathrm{B}\left(\mathrm{r}^{2}=0.65\right), \mathrm{Mg}\left(\mathrm{r}^{2}=0.57\right)$, and $\mathrm{Co}\left(\mathrm{r}^{2}=0.49\right)$, and similarly, aluminum showed a positive correlation with $\mathrm{Cd}(0.95), \mathrm{Pb}(0.79), \mathrm{Cr}$ (0.71), and Ag (0.69). High correlations were obtained between alkaline earth elements $(\mathrm{Mg}, \mathrm{Ca}, \mathrm{Sr}, \mathrm{Ba})$, which was indicated by the $\mathrm{Ca} / \mathrm{Mg}\left(\mathrm{r}^{2}=0.80\right), \mathrm{Sr} / \mathrm{Mg}\left(\mathrm{r}^{2}=0.57\right), \mathrm{Ba} / \mathrm{Mg}\left(\mathrm{r}^{2}=0.68\right), \mathrm{Ba} / \mathrm{Ca}\left(\mathrm{r}^{2}=0.86\right)$, and $\mathrm{Sr} / \mathrm{Ba}\left(\mathrm{r}^{2}=0.86\right)$ couples having significant correlations. This suggests a common source of these heavy metals from the weathering of carbonate minerals in the area [34]. 
Table 5. Trace element compositions of spring water samples (in $\mu \mathrm{g} / \mathrm{L}$ ).

\begin{tabular}{|c|c|c|c|c|c|c|c|c|c|c|c|c|c|c|c|c|c|c|}
\hline Spring ID & $\mathbf{L i}$ & B & Al & Ba & TI & $\mathrm{Pb}$ & $\mathbf{B i}$ & $\mathrm{Cr}$ & Mn & $\mathrm{Fe}$ & Co & $\mathrm{Ni}$ & $\mathrm{Cu}$ & $\mathrm{Zn}$ & $\mathrm{Sr}$ & Mo & Ag & $\mathrm{Cd}$ \\
\hline BA/085 & 1.37 & 60.8 & 6.17 & 36.3 & 0.032 & 0.21 & 0.08 & 0.51 & 0.23 & 176.1 & 0.13 & 2.26 & 1.3 & 18.28 & 124.4 & 0.20 & 0.04 & 0.008 \\
\hline $\mathrm{BA} / 085 \mathrm{~A}$ & 1.48 & 30.4 & 0.69 & 32.5 & 0.032 & 0.1 & 0.08 & 0.41 & 0.04 & 179.9 & 0.07 & 0.95 & 1.98 & 4.50 & 166.7 & 0.20 & 0.04 & 0.007 \\
\hline BA/108 & 0.76 & 26.3 & 5.51 & 13.3 & 0.032 & 0.31 & 0.08 & 0.35 & 0.2 & 97.2 & 0.06 & 0.99 & 0.71 & 3.30 & 84.40 & 0.20 & 0.04 & 0.01 \\
\hline BA/109 & 1.25 & 32.6 & 2.48 & 19.1 & 0.01 & 0.23 & 0.08 & 2.41 & 0.66 & 129.5 & 0.17 & 3.9 & 1.32 & 12.2 & 94.21 & 0.20 & 0.04 & 0.01 \\
\hline $\mathrm{BA} / 110$ & 1.41 & 30.8 & 2.06 & 29.07 & 0.03 & 0.08 & 0.08 & 0.56 & 0.14 & 106.8 & 0.09 & 2.07 & 0.36 & 2.11 & 100.7 & 0.20 & 0.04 & 0.008 \\
\hline BA/111 & 1.55 & 43.3 & 1.59 & 39.6 & 0.04 & 0.09 & 0.08 & 0.98 & 0.14 & 222.7 & 0.27 & 2.8 & 0.83 & 3.28 & 167.1 & 0.20 & 0.04 & 0.013 \\
\hline $\mathrm{BA} / 112$ & 0.91 & 27.2 & 0.69 & 13.4 & 0.03 & 0.14 & 0.08 & 0.55 & 0.22 & 105.1 & 0.06 & 5.82 & 0.54 & 4.33 & 85.44 & 0.20 & 0.04 & 0.01 \\
\hline $\mathrm{BA} / 117$ & 0.43 & 23.6 & 22.3 & 15.07 & 0.04 & 0.11 & 0.08 & 0.58 & 0.43 & 144.4 & 0.09 & 1.89 & 0.51 & 2.31 & 83.44 & 0.20 & 0.04 & 0.01 \\
\hline $\mathrm{BA} / 121$ & 0.93 & 28.5 & 11.1 & 20.07 & 0.14 & 0.46 & 0.08 & 1.78 & 0.64 & 140.1 & 0.11 & 2.42 & 0.82 & 3.50 & 131.6 & 0.20 & 0.04 & 0.03 \\
\hline $\mathrm{BA} / 122$ & 0.32 & 12.2 & 6.91 & 9.68 & 0.05 & 0.07 & 0.08 & 0.18 & 0.06 & 60.6 & 0.04 & 0.09 & 0.11 & 0.02 & 65.60 & 0.20 & 0.04 & 0.01 \\
\hline $\mathrm{BA} / 163$ & 0.27 & 10.5 & 6.52 & 6.81 & 0.04 & 0.18 & 0.08 & 0.19 & 0.13 & 70.0 & 0.03 & 1.28 & 0.11 & 0.58 & 47.40 & 0.20 & 0.04 & 0.01 \\
\hline BA/164 & 1.61 & 26.5 & 17.3 & 14.8 & 0.03 & 0.51 & 0.08 & 1.33 & 26.7 & 104.6 & 0.28 & 1.48 & 0.45 & 3.19 & 124.0 & 1.01 & 0.05 & 0.03 \\
\hline BA/170 & 0.72 & 14.5 & 6.46 & 7.75 & 0.03 & 0.51 & 0.08 & 1.33 & 0.23 & 60.9 & 0.05 & 0.65 & 0.35 & 2.32 & 49.80 & 0.66 & 0.10 & 0.03 \\
\hline BA/171 & 1.18 & 34.8 & 22.07 & 14.5 & 0.03 & 0.51 & 0.08 & 1.44 & 19.6 & 89.7 & 0.18 & 6.07 & 0.66 & 3.07 & 63.90 & 1.13 & 0.16 & 0.03 \\
\hline BA/172 & 0.75 & 17.2 & 3.34 & 7.82 & 0.03 & 0.51 & 0.08 & 1.29 & 0.21 & 72.02 & 0.03 & 0.81 & 0.34 & 2.88 & 57.10 & 0.45 & 0.12 & 0.03 \\
\hline NS/041 & 0.81 & 19.7 & 17.04 & 12.2 & 0.03 & 0.51 & 0.08 & 1.24 & 0.25 & 63.2 & 0.06 & 0.9 & 0.34 & 3.006 & 59.70 & 0.34 & 0.13 & 0.03 \\
\hline NS/007 & 1.09 & 20.1 & 110.8 & 15.04 & 0.03 & 1.07 & 0.08 & 2.97 & 3.29 & 109.7 & 0.19 & 1.37 & 0.53 & 5.48 & 80.40 & 0.54 & 0.46 & 0.12 \\
\hline NS/045 & 0.71 & 13.6 & 75.4 & 8.83 & 0.03 & 0.74 & 0.08 & 2.78 & 0.50 & 78.8 & 0.12 & 1.16 & 0.38 & 5.27 & 54.90 & 0.78 & 0.57 & 0.10 \\
\hline Ns/02 & 0.87 & 25.0 & 22.9 & 24.8 & 0.03 & 0.51 & 0.08 & 1.67 & 0.30 & 112.7 & 0.11 & 1.21 & 0.8 & 3.71 & 101.8 & 0.42 & 0.45 & 0.03 \\
\hline Ns/01 & 1.03 & 31.5 & 11.1 & 18.4 & 0.04 & 0.51 & 0.08 & 1.44 & 0.28 & 98.4 & 0.16 & 1.82 & 0.98 & 3.38 & 81.17 & 0.37 & 0.54 & 0.03 \\
\hline MIN & 0.27 & 10.5 & 0.69 & 6.81 & 0.01 & 0.07 & 0.08 & 0.18 & 0:04 & 60.6 & 0.03 & 0.09 & 0.11 & 0.02 & 47.39 & 0.2 & 0.04 & 0.007 \\
\hline MAX & 1.61 & 60.8 & 110.8 & 39.6 & 0.14 & 1.07 & 0.08 & 2.97 & 26.7 & 222.7 & 0.28 & 6.07 & 1.98 & 18.28 & 167.13 & 1.13 & 0.57 & 0.12 \\
\hline AV & 0.97 & 26.4 & 17.6 & 17.9 & 0.03 & 0.36 & 0.08 & 1.19 & 2.7 & 111 & 0.11 & 1.99 & 0.67 & 4.33 & 91.2 & 0.39 & 0.15 & 0.02 \\
\hline
\end{tabular}


Table 6. Correlation matrix of trace elements in groundwater samples.

\begin{tabular}{|c|c|c|c|c|c|c|c|c|c|c|c|c|c|c|c|c|c|c|}
\hline & $\mathrm{Mg}$ & $\mathrm{Ca}$ & $\mathbf{L i}$ & B & Al & $\mathbf{B a}$ & TI & $\mathrm{Pb}$ & $\mathrm{Cr}$ & Mn & $\mathrm{Fe}$ & Co & $\mathrm{Cu}$ & $\mathrm{Zn}$ & $\mathrm{Sr}$ & Mo & Ag & Cd \\
\hline $\mathrm{Mg}$ & 1 & & & & & & & & & & & & & & & & & \\
\hline $\mathrm{Ca}$ & 0.80 & 1 & & & & & & & & & & & & & & & & \\
\hline $\mathbf{L i}$ & 0.35 & 0.51 & 1 & & & & & & & & & & & & & & & \\
\hline Al & -0.40 & -0.35 & -0.07 & -0.27 & 1 & & & & & & & & & & & & & \\
\hline $\mathrm{Ba}$ & 0.68 & 0.86 & 0.72 & 0.82 & -0.23 & 1 & & & & & & & & & & & & \\
\hline TI & -0.05 & 0.17 & -0.14 & -0.01 & -0.07 & 0.03 & 1 & & & & & & & & & & & \\
\hline $\mathrm{Pb}$ & -0.56 & -0.59 & -0.01 & -0.28 & 0.79 & -0.38 & 0.01 & 1 & & & & & & & & & & \\
\hline Mn & -0.34 & -0.22 & 0.39 & 0.09 & 0.10 & -0.12 & -0.10 & 0.25 & 0.13 & 1 & & & & & & & & \\
\hline $\mathrm{Fe}$ & 0.75 & 0.95 & 0.61 & 0.75 & -0.15 & 0.88 & 0.14 & -0.36 & -0.09 & -0.09 & 1 & & & & & & & \\
\hline Co & 0.22 & 0.28 & 0.70 & 0.47 & 0.26 & 0.42 & -0.06 & 0.24 & 0.43 & 0.59 & 0.48 & 1 & & & & & & \\
\hline $\mathrm{Cu}$ & 0.44 & 0.66 & 0.59 & 0.64 & -0.20 & 0.67 & -0.02 & -0.20 & 0.02 & -0.10 & 0.70 & 0.23 & 1 & & & & & \\
\hline $\mathrm{Zn}$ & 0.31 & 0.37 & 0.42 & 0.70 & 0.03 & 0.45 & -0.19 & 0.0 & 0.20 & -0.08 & 0.43 & 0.24 & 0.57 & 1 & & & & \\
\hline $\mathrm{Sr}$ & 0.57 & 0.87 & 0.73 & 0.65 & -0.24 & 0.86 & 0.24 & -0.34 & -0.14 & 0.06 & 0.89 & 0.49 & 0.69 & 0.28 & 1 & & & \\
\hline Mo & -0.55 & -0.53 & 0.18 & -0.15 & 0.40 & -0.34 & -0.20 & 0.61 & 0.45 & 0.79 & -0.35 & 0.42 & -0.24 & -0.13 & -0.27 & 1 & & \\
\hline $\mathrm{Ag}$ & -0.24 & -0.41 & -0.08 & -0.21 & 0.69 & -0.15 & -0.13 & 0.72 & 0.66 & -0.06 & -0.23 & 0.18 & -0.05 & -0.02 & -0.27 & 0.35 & 1 & \\
\hline $\mathrm{Cd}$ & -0.47 & -0.44 & -0.04 & -0.32 & 0.95 & -0.29 & -0.02 & 0.89 & 0.80 & 0.11 & -0.24 & 0.25 & -0.22 & -0.01 & -0.28 & 0.48 & 0.75 & 1 \\
\hline
\end{tabular}

Bold values indicate strong positive correlation $\left(\mathrm{r}^{2} \geq 0.50\right)$ between variables. 


\subsubsection{Hierarchical Cluster Analysis (HCA)}

Hierarchical cluster analysis (HCA) was used to investigate the similarities between the various monitoring sites. In hydrological studies, R-mode cluster analysis specifically has been used to determine the association of different water quality parameters, and ultimately, the sources and processes with which they are associated [35]. In the present study, Q-mode cluster analysis was performed on the water chemistry and rock geochemistry data (major and trace elements) to group the samples according to the rock-water interactions. The results of hierarchical clustering methods depend on the specific measure of similarity and the linking method. Ward's linkage method was applied, and Euclidean distance was chosen as a measure of similarity. The results of hierarchical cluster analysis are given as a dendrogram (Figure 9a,b). Parameters belonging to the same cluster were likely to be found from the same source. As seen from this figure, the sampling sites could mainly be grouped into two clusters corresponding to the dolomite and calcite dissolution: cluster $\mathrm{A}$ and cluster B. Cluster B is subdivided into two subgroups: B-1 and B-2. The number of clusters was chosen based on the minimum number of clusters that explain most of the variation in the hydrogeochemical properties of the water and rock samples. This separation is clear in the alkaline earth elements data $(\mathrm{Mg}, \mathrm{Ca}, \mathrm{Sr}, \mathrm{Ba})$ in both the rock and groundwater samples, especially regarding Sr values. For hierarchical cluster analysis (HCA) in Q-mode applied to rock samples, classification is based on major and trace elements $(n=55)$ (Figure 9a). Cluster A consisted of BA/109, BA/164, BA/172, NS/02, NS/045, BA/170, and BA/171, which are calcite rocks according to Table 1, except for BA/109, which indicates that the calcite trace element compositions have similar characteristics and ratios. Cluster B is subdivided into two subclusters: B-1 and B-2. Cluster B-1 consisted of sites BA/171, BA/163, NS/01, and BA/112. This cluster site is close to cluster A; they have similar mineralogy properties (calcite rock) as indicated by its linkage distances. Thus, it is not surprising that cluster A and cluster B-1 were grouped together in the similar clusters. Cluster B-2 included BA/085, BA/085A, BA/110, $\mathrm{BA} / 108, \mathrm{BA} / 122$, NS/007, BA/121, NS/041, and BA/117, which reflected that the geochemistry data of this cluster is controlled by the typical geological processes (dolomite) of the study area.

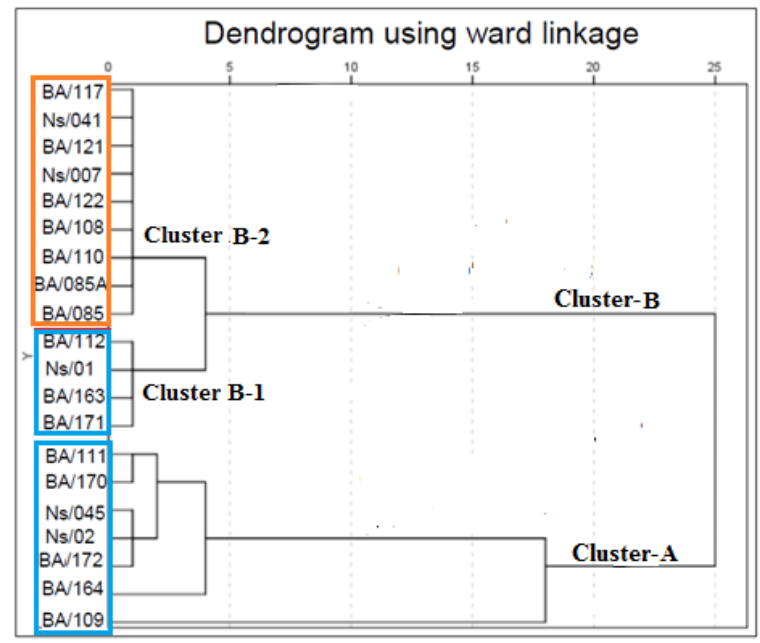

(a)

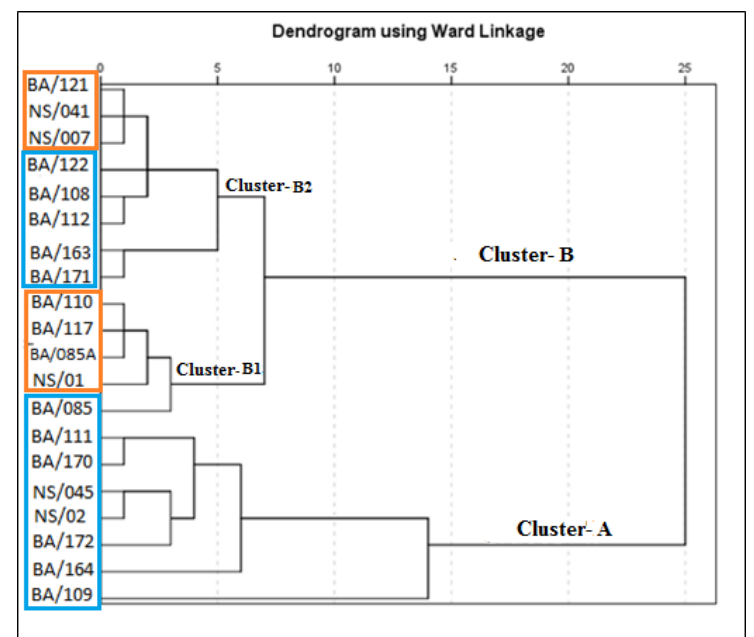

(b)

Figure 9. (a) Dendrogram resulting from the hierarchical cluster analysis (HCA) in Q-mode applied to rock samples; classification is based on major and trace elements $(n=55)$. (b) Dendrogram resulting from the HCA in Q-mode applied to groundwater samples; classification is based on major and trace elements $(n=20)$. The blue group represents the calcite dissolution, and the orange group represents the dolomite dissolution. 
R-mode cluster analysis was applied to groundwater samples; classification was based on major and trace elements $(n=20)$ to predict the rock-water interactions, and the results are shown in Figure $9 \mathrm{~b}$. Cluster A consists of BA/109, BA/164, BA/172, NS/02, NS/045, BA/170, BA/111, and BA/085, indicating that calcite dissolution dominates. Cluster $B$ was subdivided into two subclusters $B-1$ and B-2. Cluster B-1 consisted of sites NS/01, BA/085A, BA/117, and BA/110. Cluster B-2 consisted of two groups; the first group included the spring water samples BA/171, BA/163, BA/112, BA/108, and BA/122. This group represented dominant calcite dissolution. The second group consisted of NS/007, NS041, and BA/121, which indicated that dolomite dissolution dominated. Near similar groupings of parameters were observed in Figure 9a,b. The interrelationship of sediments and groundwater is visible in the two figures, indicating the geogenic hydrogeochemical evolution of groundwater by rock-water interaction. Such a grouping pattern signifies the strength of their mutual relation, which suggests that the weathering of rocks primarily controls the major ion chemistry of groundwater in this region. The dispersion of calcite and dolomite samples in Figure $9 \mathrm{~b}$ indicated the effect of anthropogenic activities on the chemistry of groundwater in the wet season, indicating that the dissolution of carbonate rocks (calcite, dolomite) was not the only source for these elements in the rainy season.

\section{Conclusions}

This research is one of the first mineralogical and geochemical analyses of sediments from the Central West Bank. New data on the mineralogy and geochemistry for the sedimentary rocks of the CWB were used for an integrated study to clarify the geological control of the groundwater system, identify the natural weathering processes within the catchment, and establish a link between the groundwater hydrochemistry and geological materials. The results overall suggested that the composition of groundwater in the CWB was mainly influenced by the source lithology and geology characteristics of the study area. The mineralogical composition of 20 rock samples was obtained using the X-ray diffraction technique (XRD) with a semi-quantitative determination of the minerals. The studied samples were highly homogenous in terms of mineralogical composition, with a predominance of calcite and dolomite, with minor amounts of feldspars and quartz. The multivariate statistical analyses allowed for more detailed and comparable results complementing to the traditional hydrochemical and geochemical analyses methods. The HCA has classified the groundwater and sediment samples into two major clusters: a calcite group and a dolomite group.

Author Contributions: H.J. gathered the data, which were evaluated in consultation with all authors. The manuscript was written by H.J. and reviewed by all authors.

Funding: This research received partial funding from the department of hydrogeology (Ruhr University Bochum).

Acknowledgments: We acknowledge support by the Open Access Publication Funds of the Ruhr University Bochum. The authors thank Oliver Schübbe and Thomas Reinecke (Ruhr University Bochum) and Mutaz Al-Qutob (Al-Quds University) for analytical work. The authors gratefully acknowledge two anonymous reviewers for their critical evaluation and suggestions, which greatly helped to improve the manuscript.

Conflicts of Interest: The authors declare no conflict of interest.

\section{References}

1. Jeannina, P.Y.; Hessenauer, M.; Malard, A.; Chapuis, V. Impact of global change on karst groundwater mineralization in the Jura Mountains. Sci. Total Environ. 2016, 541, 1208-1221. [CrossRef] [PubMed]

2. Bakalowicz, M. Karst and karst groundwater resources in the Mediterranean. Environ. Earth Sci. 2015, 74, 5-14. [CrossRef]

3. Galvão, P.; Halihan, T.; Hirata, R. Evaluating karst geotechnical risk in the urbanized area of Sete Lagoas, Minas Gerais, Brazil. Hydrogeol. J. 2015, 23, 1499-1513. [CrossRef]

4. Jebreen, H.; Wohnlich, S.; Banning, A.; Wisotzky, F.; Niedermayr, A.; Ghanem, M. Recharge, geochemical processes and water quality in karst aquifers: Central West Bank, Palestine. Environ. Earth Sci. 2018, 77, 261. [CrossRef] 
5. Zwahlen, F. Vulnerability and Risk Mapping for the Protection of Carbonate (Karst) Aquifers, Final Report COST Action 620; European Commission: Brussels, Belgium, 2004.

6. Foster, S.; Hirata, R.; Andreo, B. The aquifer pollution vulnerability concept: Aid or impediment in promoting groundwater protection? Hydrogeol. J. 2013, 21, 1389-1392. [CrossRef]

7. Siebert, C.; Rosenthal, P.; Möller, P.; Rödiger, T.; Meiler, M. The hydrochemical identification of groundwater flowing to the Bet She'an-Harod multiaquifer system (Lower Jordan Valley) by rare earth elements, yttrium, stable isotopes (H, O) and Tritium. Appl. Geochem. 2012, 27, 703-714. [CrossRef]

8. Kronfeld, J.; Rosenthal, E. In search of a characteristic signature for groundwater aquifers-A case study from Israel-Comment. J. Hydrol. 1987, 93, 359-372. [CrossRef]

9. Weinberger, G.; Rosenthal, E.; Ben-Zvi, A.; Zeitoun, D.G. The Yarkon-Taninim groundwater basin, Israel hydrogeology: Case study and critical review. J. Hydrol. 1994, 161, 227-255. [CrossRef]

10. Rofe and Raffety Consulting Engineers. Nablus District Water Resources Survey; Geological and Hydrological Report; Hashemite Kingdom of Jordan, Central Water Authority, Westminster: London, UK, 1965.

11. Abed, A.; Wishahi, S. Geology of Palestine: The West Bank and Gaza Strip; Palestinian Hydrology Group: Jerusalem, Israel, 1999.

12. Jebreen, H.; Wohnlich, S.; Wisotzky, F.; Banning, A.; Niedermayr, A.; Ghanem, M. Recharge estimation in semi-arid karst catchments: Central West Bank, Palestine. Grundwasser J. 2018, 23, 91-101. [CrossRef]

13. Abusaada, M.; Sauter, M. Studying the flow dynamics of a karst aquifer system with an equivalent porous medium model. Groundwater 2013, 51, 641-650. [CrossRef]

14. Sheffer, N.A.; Cohen, M.; Morin, E.; Grodek, T.; Gimburg, A.; Magal, E.; Gvirtzman, H.; Nied, M.; Isele, D.; Frumkin, A. Integrated cave drip monitoring for epikarst recharge estimation in a dry Mediterranean area, Sif Cave, Israel. Hydrol. Process. 2011, 25, 2837-2845. [CrossRef]

15. Dafny, E.; Burg, A.; Gvirtzman, H. Effects of Karst and geological structure on groundwater flow: The case of Yarqon-Taninim Aquifer, Israel. J. Hydrol. 2010, 389, 260-275. [CrossRef]

16. Weiss, M.; Gvirtzman, H. Estimating ground water recharge using flow models of perched Karstic aquifers. Groundw. J. 2007, 45, 761-773. [CrossRef] [PubMed]

17. Lange, J.; Greenbaum, N.; Husary, S.; Ghanem, M.; Leibundgut, C.; Schick, A.P. Runoff generation from successive simulated rainfalls on a rocky, semi-arid, Mediterranean hillslope. Hydrol. Process. 2003, 17, 279-296. [CrossRef]

18. SUSMAQ. Sustainable Management of the West Bank and Gaza Aquifers: Monthly Recharge Estimation in Wadi Natuf, Palestine; Palestinian Water Authority, Palestine and the University of Newcastle upon Tyne: Newcastle, UK, 2004.

19. Issar, A.S. Recharge and salination processes in the carbonate aquifers in Israel. Environ. Geol. 1993, 21, 152-159. [CrossRef]

20. Palestinian Water Authority (PWA). Hydrology Department; PWA: Ramallah, Israel, 2016.

21. Abed Rabbo, A.; Scarpa, D.J.; Qannam, Z.; Abdul Jaber, Q.; Yaunger, P. Springs in the West Bank—Water Quality and Chemistry; Palestinian Hydrology Group and Newcastle University, CMS Ltd.: Palestine, Israel, 1999.

22. Frumkin, A. Interaction between karst, water and agriculture over the climatic gradient of Israel. Int. J. Speleol. 1999, 26B, 99-110. [CrossRef]

23. Avisar, D.; Rosenthal, E.; Flexer, A.; Shulman, H.; Ben-Avraham, Z.; Guttman, J. Salinity sources of Kefar Uriya wells in the Judea Group aquifer of Israel. Part 1, conceptual hydrogelogical model. J. Hydrol. 2003, 207, 27-38. [CrossRef]

24. Gromet, L.P.; Dymek, R.F.; Haskin, L.A.; Korotev, R.L. The "North American shale composite": Its compilation, major and trace element characteristics. Geochim. Cosmochim. Acta 1984, 48, 2469-2482. [CrossRef]

25. Malassa, H.; Al-Rimawi, F.; Al-Khatib, M.; Al-Qutob, M. Determination of trace heavy metals in harvested rainwater used for drinking in Hebron (south West Bank, Palestine) by ICP-MS. Environ. Monit. Assess. J. 2014, 186, 6985-6992. [CrossRef]

26. Clark, E.V.; Daniels, W.L.; Zipper, C.E.; Eriksson, K. Mineralogical influences on water quality from weathering of surface coal mine spoils. Appl. Geochem. 2018, 91, 97-106. [CrossRef]

27. Taylor, S.R.; McLennan, S.M. The Continental Crust: Its Composition and Evolution; Blackwell Scientific Publication: Carlton, Australia, 1985; 312p. 
28. Yang, X.; Mei, Q.; Wang, X.; Dong, Z.; Li, Y.; Huo, F. Indication of rare earth element characteristics to dolomite petrogenesis-A case study of the fifth member of Ordovician Majiagou Formation in the Ordos Basin, central China. Mar. Pet. Geol. 2018, 92, 1028-1040. [CrossRef]

29. Worrall, F.; Pearson, D.G. Water-rock interaction in an acidic mine discharge as indicated by rare earth element patterns. Geochim. Cosmochim. Acta 2001, 65, 3027-3040. [CrossRef]

30. Ford, D.C.; Williams, P.W. Karst Hydrogeology and Geomorphology; Wiley: Chichester, UK, 2007.

31. Piper, A.M. A graphic procedure in the geochemical interpretation of water analyses. Am. Geophys. Union Trans. 1944, 25, 914-928. [CrossRef]

32. Nekouei, E.; Zarei, M.; Raeisi, E. The influence of diapir brine on groundwater quality of surrounding aquifers, Larestan, Iran. Environ. Earth Sci. 2016, 75, 571. [CrossRef]

33. Ma, R.; Wang, Y.; Sun, Z.; Zheng, C.; Ma, T.; Prommer, H. Geochemical evolution of groundwater in carbonate aquifers in Taiyuan, northern China. Appl. Geochem. 2011, 26, 884-897. [CrossRef]

34. Gascoyne, M. Trace-element partition coefficients in the calcite-Water system and their paleoclimatic significance in cave studies. J. Hydrol. 1983, 61, 213-222. [CrossRef]

35. Drew, L.; Grunsky, E.; Sutphin, D.; Woodruff, L. Multivariate analysis of the geochemistry and mineralogy of soils along two continental-scale transects in North America. Sci. Total Environ. 2010, 409, $218-227$. [CrossRef]

(C) 2018 by the authors. Licensee MDPI, Basel, Switzerland. This article is an open access article distributed under the terms and conditions of the Creative Commons Attribution (CC BY) license (http:/ / creativecommons.org/licenses/by/4.0/). 